\title{
Analysis of Xanthomonas oryzae pv. oryzicola Population in Mali and Burkina Faso Reveals a High Level of Genetic and Pathogenic Diversity
}

\author{
I. Wonni, B. Cottyn, L. Detemmerman, S. Dao, L. Ouedraogo, S. Sarra, C. Tekete, S. Poussier, R. Corral, \\ L. Triplett, O. Koita, R. Koebnik, J. Leach, B. Szurek, M. Maes, and V. Verdier
}

First, twelfth, fourteenth, and sixteenth authors: Institut de Recherche pour le Développement, UMR Résistance des Plantes aux Bioagresseurs, IRD-CIRAD-UM2, 911 Avenue Agropolis BP 64501, 34394 Montpellier Cedex 5, France; first and fifth authors: Institut de l'Environnement et de Recherches Agricoles (INERA), 01 B.P. 910 Bobo Dioulasso, Burkina Faso; second, third, and fifteenth authors: Plant Sciences Unit-Crop Protection, Institute for Agricultural and Fisheries Research (ILVO), Burg. van Gansberghelaan 96, 9820 Merelbeke, Belgium; fourth, seventh, and eleventh authors: University Bamako, FAST Laboratoire Biologie Moléculaire Appliquée (LBMA), Bamako, Mali; sixth author: Institut d'Economie Rural, Niono, Mali; eighth author: Université de La Réunion, UMR 53 Peuplements Végétaux et Bioagresseurs en Milieu Tropical (PVBMT), 15 avenue René Cassin, BP 7151, F-97715 Saint-Denis Cedex 9, Réunion, France; and ninth, tenth, thirteenth, and sixteenth authors: Bioagricultural Sciences, Colorado State University, Plant Sciences Bldg., Fort Collins 80523-1177.

Accepted for publication 27 October 2013.

\begin{abstract}
Wonni, I., Cottyn, B., Detemmerman, L., Dao, S., Ouedraogo, L., Sarra, S., Tekete, C., Poussier, S., Corral, R., Triplett, L., Koita, O., Koebnik, R., Leach, J., Szurek, B., Maes, M., and Verdier, V. 2014. Analysis of Xanthomonas oryzae pv. oryzicola population in Mali and Burkina Faso reveals a high level of genetic and pathogenic diversity. Phytopathology 104:520-531.

Bacterial leaf streak (BLS) caused by Xanthomonas oryzae pv. oryzicola was first reported in Africa in the 1980s. Recently, a substantial reemergence of this disease was observed in West Africa. Samples were collected at various sites in five and three different rice-growing regions of Burkina Faso and Mali, respectively. Sixty-seven X. oryzae pv. oryzicola strains were isolated from cultivated and wild rice varieties and from weeds showing BLS symptoms. X. oryzae pv. oryzicola strains were evaluated for virulence on rice and showed high variation in lesion length

characterized by multilocus sequence analysis (MLSA) using six housekeeping genes. Inferred dendrograms clearly indicated different groups among $X$. oryzae pv. oryzicola strains. Restriction fragment length polymorphism analysis using the transcriptional activator like effector avrXa7 as probe resulted in the identification of 18 haplotypes. Polymerase chain reaction-based analyses of two conserved type III effector (T3E) genes (xopAJ and $x o p W)$ differentiated the strains into distinct groups, with xopAJ not detected in most African X. oryzae pv. oryzicola strains. XopAJ functionality was confirmed by leaf infiltration on 'Kitaake' rice Rxol lines. Sequence analysis of xop $W$ revealed four groups among $X$. oryzae pv. oryzicola strains. Distribution of $43 \mathrm{~T} 3 \mathrm{E}$ genes shows variation in a subset of $X$. oryzae pv. oryzicola strains. Together, our results show that African $X$. oryzae pv. oryzicola strains are diverse and rapidly evolving, with a group endemic to Africa and another one that may have evolved from an Asian strain.
\end{abstract} on a susceptible cultivar. X. oryzae pv. oryzicola strains were further
Rice cultivation has greatly increased during the last decade in West Africa. In Mali and Burkina Faso, initiatives are underway to expand rice cultivation and increase local production; intensification increases the risk for the emergence of rice pathogens such as Xanthomonas oryzae $(27,45)$. X. oryzae causes two important diseases of rice: $X$. oryzae pv. oryzae colonizes xylem vessels and causes bacterial leaf blight, whereas $X$. oryzae pv. oryzicola colonizes spaces between leaf parenchyma cells to cause bacterial leaf streak (BLS). BLS has been reported in several tropical and subtropical Asian countries, including Thailand, Malaysia, India, Vietnam, the Philippines, and Indonesia $(26,30,39,40)$. In Africa, the disease was reported in Madagascar, Senegal, and Nigeria in the 1980s (5) and emerged more recently in Mali and Burkina Faso $(13,49)$. Due to changing agricultural practices and the recent introduction of susceptible rice varieties,

Corresponding author: V. Verdier; E-mail address: valerie.verdier@ird.fr

* The $\boldsymbol{e}$-Xtra logo stands for "electronic extra" and indicates that the online version contains one supplemental table and two supplemental figures. Figures 2, 4, and 6 appear in color online.

http://dx.doi.org/10.1094/PHYTO-07-13-0213-R

(C) 2014 The American Phytopathological Society
BLS has been increasing in significance in China and Africa $(13,48,49,51)$. BLS may affect entire fields and cause yield losses up to $32 \%(21,50,51)$. BLS is included in the quarantine lists of most rice-growing countries.

The species $X$. oryzae is highly diverse, represented by distinct phylogenetic clades for $X$. oryzae pv. oryzae from Asia, Africa, and the United States, and for $X$. oryzae pv. oryzicola from Asia and Africa (46). X. oryzae pv. oryzae and X. oryzae pv. oryzicola are genetically and phenotypically highly related and, therefore, difficult to differentiate (34). Although $X$. oryzae pv. oryzae and $X$. oryzae pv. oryzicola infect their host in distinct ways and the symptoms provoked are different at an early stage of infection, the two diseases are not easy to distinguish at a later stage (28). BLS symptoms occur as small, translucent, and typically yellow lesions along the leaf between the veins that expand lengthwise. At later stages, leaves turn grayish white and die. X. oryzae pv. oryzicola exudes from natural openings in the leaf, and strings of yellow exudates along young lesions on the leaf surface are typical of BLS.

Despite the importance of BLS, few population studies of the pathogen have been conducted. In the Philippines and in southern of China, X. oryzae pv. oryzicola strains were collected from 
different rice-growing regions $(31,35)$. In Africa, only a few $X$. oryzae pv. oryzicola strains were isolated and characterized $(13,49)$. Studies relying on DNA fingerprinting methods such as restriction fragment length polymorphisms (RFLP), polymerase chain reaction (PCR) using rep elements, and amplified fragment length polymorphism (AFLP) revealed variability among $X$. oryzae pv. oryzicola strains with no correlation to either sampling date or location, or to host reaction $(31,35)$. African $X$. oryzae pv. oryzicola strains were found that are genetically closely related to Asian strains (13). A recent multilocus sequence analysis (MLSA) study highlighted substantial differences among strains of the $X$. oryzae species $(17,46)$. MLSA did not distinguish among $X$. oryzae pv. oryzicola strains; however, the low number of strains analyzed limited conclusions (17). Recently, multiple loci variable number of tandem repeat (VNTR) analysis (MLVA) was performed on a diverse but limited collection of $X$. oryzae pv. oryzicola strains isolated in 2008 and 2009, including a few strains from West Africa (56). MLVA confirmed that Asian $X$. oryzae pv. oryzicola strains are distinct from African ones and identified a possible origin of some Asian strains in Africa (17).

Recent acquisition of genomic and genetic resources for $X$. oryzae pv. oryzicola has enabled the identification of virulence factors $(6,57)$. X. oryzae pv. oryzicola encodes a large number of transcription activator-like effector (TALE) genes, with up to 26 copies in the genome (6). Some of them contribute as potential virulence factors (R. A. Cernadas, L. Wang, and A. J. Bogdanove, unpublished data) (25). Guo et al. (15) identified new candidate genes involved in $X$. oryzae pv. oryzicola virulence, including a TALE gene.

Little information is available on virulence characteristics and genetic variability of $X$. oryzae pv. oryzicola in Africa. Virulence of $X$. oryzae pv. oryzicola strains is known to be highly variable, although no clear differential host-strain interaction is defined yet $(3,13)$. No single rice resistance gene source is available to control BLS disease, forcing breeding programs to focus on quantitative sources of resistance $(9,18,44)$. Rxol, a resistance $(R)$ gene identified from maize, encodes an intracellular nucleotide-binding leucine-rich repeat $\mathrm{R}$ protein and confers immunity against $X$. oryzae containing the effector gene xopAJ (synonym avrRxol) when expressed in rice $(54,55)$. Although the type III effector (T3E) content is very similar among Asian and African $X$. oryzae pv. oryzicola strains, the T3E gene $x o p A J$ is absent in most of the African strains analyzed thus far $(13,17,54)$.

Given the emergence of BLS worldwide and increased exchange of rice seed in Africa, it is imperative that a broader and deeper understanding of the structure of West African $X$. oryzae pv. oryzicola populations is developed. This is an essential prerequisite to guide selection and the rational deployment of BLS resistance.

The objectives of this study were to (i) characterize the pathogenicity of $X$. oryzae pv. oryzicola strains recently isolated in Mali and Burkina Faso and (ii) determine the genetic diversity of these strains using MLSA and other DNA-based methods.

\section{MATERIALS AND METHODS}

Collection of strains. X. oryzae pv. oryzicola strains were obtained from different origins and collections (Table 1). Surveys for rice BLS disease were performed in 2009, 2010, and 2011 in major rice production areas in Mali and Burkina Faso. Field visits were conducted in September to October of each year during the rainy season when symptoms were visible. Leaves with BLS-like symptoms (translucent, yellow streaks, sometimes with visible bacterial exudates on the leaf surface) were collected from Oryza sativa cultivars, wild rice species, and weeds. Bacterial isolations were performed as previously described (1). Briefly, sections from the diseased leaf tissue were disinfected and macerated in sterile distilled water. The macerate was diluted and streaked onto plates of peptone-sucrose agar (PSA) medium (22) supplemented with actidione at $50 \mathrm{mg} \mathrm{liter}{ }^{-1}$, cephalexin at $40 \mathrm{mg} \mathrm{liter}^{-1}$, and kasugamycin at $20 \mathrm{mg} \mathrm{liter}^{-1}$. Plates were incubated for 2 to 5 days at $28^{\circ} \mathrm{C}$. A single bacterial colony was cultured from each tissue sample. Isolates were maintained in $15 \%$ glycerol at $-80^{\circ} \mathrm{C}$.

Multiplex PCR. All X. oryzae pv. oryzicola strains were identified using a multiplex PCR specific for $X$. oryzae pathovars (23). For DNA extraction, bacteria were grown overnight in nutrient broth with shaking at $28^{\circ} \mathrm{C}$. DNA was isolated from each strain using the Wizard Genomic DNA Purification Kit according to the manufacturer's recommendations (Promega Corporation, Madison, WI). PCR with the $X$. oryzae-specific multiplex primer was performed as previously described (23). This multiplex PCR assay directs the amplification of three fragments $(\approx 945,691$, and $324 \mathrm{bp}$ ) from $X$. oryzae pv. oryzicola strains. All isolates were tested at least twice.

Pathogenicity assays. Pathogenicity tests were performed using leaf infiltration on greenhouse-grown 3-week-old 'Nipponbare', 'Kitaake', and Kitaake rice plants harboring the Rxol resistance gene (Kitaake-Rxol). Each strain was grown on PSA for $24 \mathrm{~h}$ and then suspended in sterile deionized water. Bacterial suspensions were infiltrated with a needleless syringe into the intercellular spaces of rice leaves at concentrations of $10^{8} \mathrm{CFU}$ $\mathrm{ml}^{-1}$, as previously described (36). At least two infiltrations were done per isolate per leaf and two leaves were inoculated per plant. Negative control plants were infiltrated with sterile distilled water. Experiments were repeated three independent times. Plants were maintained in the greenhouse (day and night temperatures of $27 \pm$ $1{ }^{\circ} \mathrm{C}, 12$-h day length, and relative humidity of $80 \%$ ). The hypersensitive reaction (HR), visible as browning in response to Rxol, was scored at $72 \mathrm{~h}$ after inoculation (hai). The plants were observed regularly and lesions were scored and measured 12 days postinoculation (dpi).

MLSA. In total, 54 strains were selected for MLSA, including 41 representative African $X$. oryzae pv. oryzicola isolates collected from different rice-growing regions in Mali and Burkina Faso in 2003, 2009, 2010, and 2011; three Asian X. oryzae pv. oryzicola strains (BLS256, LMG797, and X. oryzae pv. oryzicola China); X. campestris pv. leersiae strain NCPPB4346; and nine $X$. oryzae pv. oryzae strains from different geographical origins (Africa, Asia, and the United States). Six housekeeping genesatpD (ATP synthase $\beta$ chain), dnaK (chaperone protein), efP (elongation factor P), gyrB (DNA gyrase subunit B), lepA (GTPbinding protein), and $r p o D$ (RNA polymerase $\sigma-70$ factor) -were amplified with primers listed in Table 2 , then sequenced $(4,8$, $33,52)$. The sequences of all alleles were deposited in GenBank under accession numbers JX682633 to JX682665, JX886813 to JX887059, and JX897606 to JX897675. Additional sequences were retrieved from the complete genome sequences of the Asian $X$. oryzae pv. oryzicola strain BLS256 (National Center for Biotechnology Information [NCBI] reference sequence NC_017267.1) and $X$. oryzae pv. oryzae strains MAFF311018 (NC_007705), PXO99 $^{A}$ (NC_010717), and KACC10331 (NC_006834). X. vasicola pv. vasculorum (NCPPB702) was used as an out-group in the gyrB phylogenetic analysis of the $X$. oryzae strains using sequences retrieved from GenBank (ACHS00000000.1).

The nucleotide gene sequences of each individual gene were aligned using the ClustalW algorithm and afterward trimmed in frame to obtain equal lengths. Sequence alignment, trimming, nucleotide translation into amino acid sequences, and phylogenetic analysis was performed in MEGA 5 (43). Phylogenetic trees for each independent gene alignment were constructed using the maximum composite likelihood method for calculating distances and the neighbor joining (NJ) algorithm for clustering. Phylogenetic analysis for the corresponding amino acid sequences was performed using the p-distance method and NJ clustering. For all phylogenetic trees, bootstrap analysis was performed using 1,000 bootstrap replicates. For the aligned concatenated sequences 
$(3,813 \mathrm{bp})$, a consensus split network was constructed in BioNumerics (version 6.6; Applied Maths, Belgium) using the Hamming-distance, NJ and bootstrap analysis with 1,000 resamplings.

RFLP analysis of TALE haplotypes. TALE haplotypes were determined on 53 strains using a 725-bp C-terminal avrXa7 PCR fragment as a probe, which was generated using primers Tal-CtF2 (5'-GCGTTGGCCGCGTTGACCAA-3') and Tal-Ct-R2 (5'-
GGGGCCGCATCTTGTTCCCA-3') and plasmid pH81 as DNA template (19). X. oryzae pv. oryzicola strains MAI3, MAI10, and BLS256 and $X$. campestris pv. leersiae strain NCPPB4346 were included in the analysis. For each strain, $5 \mu \mathrm{g}$ of total DNA was digested to completion with the enzyme BamHI (New England Biolabs Inc., Saint Quentin, France). Digested DNA was separated on $1 \%$ agarose gels in $1 \times$ Tris-borate EDTA buffer at $50 \mathrm{~V}$ for $72 \mathrm{~h}$. Fragments were visualized by UV radiation $(302 \mathrm{~nm})$

TABLE 1. List of strains characterized in this study

\begin{tabular}{|c|c|c|c|c|c|c|c|c|c|c|c|c|c|}
\hline Strain & Other names ${ }^{v}$ & Origin & Region & Site & Year & Host & xорAJ ${ }^{\mathrm{w}}$ & xopW ${ }^{x}$ & $\operatorname{gyrB}^{y}$ & MLSA & Vir. $^{z}$ & TAL & Reference \\
\hline \multicolumn{14}{|c|}{$X$. oryzae pv. oryzicola } \\
\hline BAI 5 & $\begin{array}{l}\text { LMG25975, } \\
\text { CFBP7341 }\end{array}$ & Burkina & Hauts Bassins & Vallée du Kou & 2009 & O. sativa & - & $>$ & II & $\mathrm{b}$ & 4 & 11 & 44 \\
\hline BAI 6 & LMG26385 & Burkina & Hauts Bassins & Vallée du Kou & 2009 & O. sativa & + & + & I & $\mathrm{c}$ & 5 & 3 & 44 \\
\hline BAI 7 & LMG26386 & Burkina & East center & Itenga & 2009 & O. longistaminata & - & + & I & $\mathrm{e}$ & 4 & 13 & This study \\
\hline BAI 8 & & Burkina & East center & Itenga & 2009 & O. longistaminata & - & + & na & - & 4 & 12 & This study \\
\hline BAI 9 & & Burkina & East center & Itenga & 2009 & O. longistaminata & - & + & na & - & 1 & 11 & This study \\
\hline BAI 10 & & Burkina & East center & Itenga & 2009 & O. longistaminata & - & + & na & - & 2 & 12 & This study \\
\hline BAI 11 & $\begin{array}{l}\text { LMG25976, } \\
\text { CFBP7342 }\end{array}$ & Burkina & East center & Itenga & 2009 & Wild rice & - & + & I & $\mathrm{f}$ & 5 & 2 & This study \\
\hline BAI 12 & LMG26387 & Burkina & East center & Bagré & 2009 & O. sativa & - & + & III & $\mathrm{a}$ & 2 & 12 & This study \\
\hline BAI 13 & LMG26388 & Burkina & East center & Bagré & 2009 & Weeds & - & + & III & $\mathrm{a}$ & 4 & 12 & This study \\
\hline BAI 14 & LMG26389 & Burkina & East center & Bagré & 2009 & O. sativa & - & + & III & $\mathrm{a}$ & 2 & 12 & This study \\
\hline BAI 15 & $\begin{array}{l}\text { LMG25977, } \\
\text { CFBP7343 }\end{array}$ & Burkina & East center & Bagré & 2009 & O. sativa & - & + & III & $\mathrm{a}$ & 3 & 7 & 44 \\
\hline BAI 16 & LMG26390 & Burkina & East center & Bagré & 2009 & O. sativa & - & + & III & $\mathrm{a}$ & 4 & 12 & This study \\
\hline BAI 17 & LMG26391 & Burkina & East center & Bagré & 2009 & Pennisetum & - & + & III & $\mathrm{a}$ & 3 & 12 & This study \\
\hline BAI 18 & & Burkina & East center & Koulwooko & 2009 & O. sativa & - & + & na & - & 3 & 2 & This study \\
\hline BAI 19 & LMG26392 & Burkina & Cascades & Karfiguela & 2009 & O. sativa & + & + & I & $\mathrm{c}$ & 5 & 11 & 44 \\
\hline BAI 20 & LMG26393 & Burkina & Cascades & Karfiguela & 2009 & Weeds & - & $>$ & II & $\mathrm{b}$ & 3 & 13 & 44 \\
\hline BAI 21 & LMG26394 & Burkina & Cascades & Karfiguela & 2009 & O. sativa & - & + & I & $\mathrm{h}$ & 2 & 3 & This study \\
\hline BAI 22 & LMG26395 & Burkina & Cascades & Karfiguela & 2009 & O. longistaminata & - & + & I & $\mathrm{f}$ & 2 & 3 & This study \\
\hline BAI 34 & & Burkina & Cascades & Karfiguela & 2009 & O. sativa & + & + & na & - & 3 & 12 & This study \\
\hline BAI 35 & LMG27225 & Burkina & Center & Nabadougou & 2010 & O. longistaminata & + & + & I & $\mathrm{c}$ & 3 & 12 & This study \\
\hline BAI 36 & LMG27226 & Burkina & B. Mouhoun & Niassan & 2010 & $O$ sativa & - & + & I & $\mathrm{h}$ & 3 & 3 & This study \\
\hline BAI 37 & & Burkina & East center & Bagré & 2010 & O. sativa & - & + & na & - & 3 & 3 & This study \\
\hline BAI 38 & & Burkina & Cascades & Karfiguela & 2009 & O. sativa & - & + & na & - & 0 & na & This study \\
\hline BAI 39 & & Burkina & Cascades & Karfiguela & 2009 & O. sativa & - & + & na & - & 3 & na & This study \\
\hline BAI 40 & & Burkina & East center & Bagré & 2010 & O. sativa & - & + & na & - & 3 & na & This study \\
\hline BAI 41 & & Burkina & Center & Nabadougou & 2010 & O. longistaminata & - & + & na & - & 3 & 8 & This study \\
\hline BAI 42 & & Burkina & East center & Bagré & 2010 & O. sativa & - & + & na & - & 3 & na & This study \\
\hline BAI 43 & LMG27227 & Burkina & Hauts Bassins & Nienadonkele & 2010 & O. sativa & + & + & I & $\mathrm{i}$ & 4 & 3 & This study \\
\hline BAI 44 & LMG27228 & Burkina & Hauts Bassins & Nienadonkele & 2010 & O. sativa & + & + & I & $\mathrm{i}$ & 4 & 3 & This study \\
\hline BAI 49 & & Burkina & B. Mouhoun & Niassan & 2011 & O. longistaminata & + & $>$ & II & $\mathrm{b}$ & 2 & na & This study \\
\hline BAI 60 & & Burkina & B. Mouhoun & Niassan & 2011 & O. sativa & - & + & I & $\mathrm{g}$ & 1 & na & This study \\
\hline BAI 62 & & Burkina & B. Mouhoun & Niassan & 2011 & Brachiaria lata & - & + & I & $\mathrm{i}$ & 3 & na & This study \\
\hline BAI 65 & & Burkina & B. Mouhoun & Niassan & 2011 & $\begin{array}{l}\text { Paspalum } \\
\text { vaginatum }\end{array}$ & - & + & I & $\mathrm{i}$ & 3 & na & This study \\
\hline BAI 68 & & Burkina & B. Mouhoun & Niassan & 2011 & O. longistaminata & - & + & I & $\mathrm{g}$ & 2 & na & This study \\
\hline BAI 69 & & Burkina & Cascades & Karfiguela & 2011 & O. longistaminata & + & $>$ & II & $\mathrm{b}$ & 2 & na & This study \\
\hline BAI 76 & & Burkina & Cascades & Karfiguela & 2011 & O. sativa & - & + & I & $\mathrm{i}$ & 2 & na & This study \\
\hline BAI 82 & & Burkina & Cascades & Karfiguela & 2011 & O. sativa & - & $>$ & II & $\mathrm{b}$ & 1 & na & This study \\
\hline MAI 16 & LMG26396 & Mali & O. Niger & Molodo & 2009 & O. sativa & + & + & I & $\mathrm{c}$ & 4 & 1 & This study \\
\hline MAI 17 & LMG26397 & Mali & O. Niger & Kogoni & 2009 & O. sativa & - & $>$ & II & $\mathrm{b}$ & 2 & 14 & This study \\
\hline MAI 18 & $\begin{array}{l}\text { LMG25981, } \\
\text { CFBP7338 }\end{array}$ & Mali & O. Niger & Kogoni & 2009 & O. longistaminata & - & + & I & $\mathrm{d}$ & 1 & 11 & This study \\
\hline MAI 19 & & Mali & O. Niger & Kogoni & 2009 & O. longistaminata & - & + & na & - & 3 & 11 & This study \\
\hline MAI 20 & LMG26398 & Mali & O. Niger & Kogoni & 2009 & O. longistaminata & + & + & I & $\mathrm{f}$ & 3 & 11 & This study \\
\hline MAI 21 & & Mali & O. Niger & Kogoni & 2009 & O. longistaminata & - & $>$ & na & - & 1 & 14 & This study \\
\hline MAI 22 & LMG26399 & Mali & O. Niger & Kogoni & 2009 & O. barthii & + & + & I & $\mathrm{f}$ & 3 & 6 & This study \\
\hline MAI 23 & $\begin{array}{l}\text { LMG25982, } \\
\text { CFBP7339 }\end{array}$ & Mali & O. Niger & Kogoni & 2009 & O. barthii & - & $>$ & II & $\mathrm{b}$ & 2 & 14 & This study \\
\hline
\end{tabular}

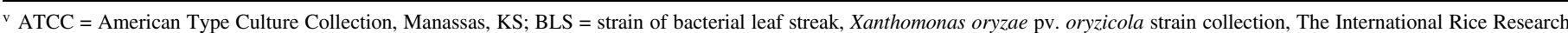
Institute (IRRI), Los Baños, Philippines; CFBP, Collection Française de Bactéries Phytopathogènes, Institut National de la Recherche Agronomique (INRA), Angers, France; LMG/BCCM = Belgian Coordinated Collection of Microorganisms, University of Gent, Belgium; NCPPB, National Collection of Plant Pathogenic Bacteria, UK; MAI, and BAI, collection of $X$. oryzae pv. oryzae and pv. oryzicola, Institut de la Recherche pour le Développement (IRD), Montpellier, France; Laboratoire de Biologie Moléculaire Appliquée (LBMA), Bamako, Mali, and Institut de l'Environnement et de Recherches Agricoles Burkina Faso (INERA); DNA of the strains X. oryzae pv. oryzicola China, PXO99A, MAFF311018 and KACC10331 were provided by J. Leach (Colorado State University, Fort Collins, CO).

${ }^{\mathrm{w}}$ Presence or absence of $x o p A J$ gene as described in the text.

${ }^{x}$ Presence or absence of $x o p W$; $>$ indicates that a DNA arrangement (insertion element) was identified within the $x o p W$ gene.

y gyrB group according to Figure 3 .

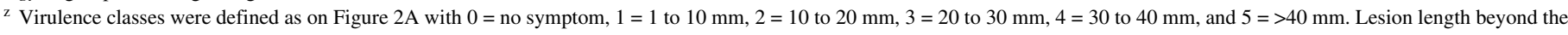
circular infiltration site was measured at 14 days after inoculation of Oryza sativa 'Nipponbare'; na = data not available. 
after staining agarose gels in ethidium bromide $(0.5 \mu \mathrm{g} / \mathrm{ml})$. Hybridization assays were performed as described previously (53). Blots were exposed on a phosphorImager (Molecular Dynamics Storm; GE Healthcare Life Sciences, France). Each strain was analyzed at least twice, and the banding patterns were compared in a single blot to confirm that each pattern was unique. Banding patterns of hybridization were used to compare the relatedness of strains. Each band was coded as 1 (presence) or 0 (absence). BioNumerics (version 6.6; Applied Math Inc., Belgium) was used for the TALE analysis and phylogenetic construction based on a minimum spanning tree (MST) algorithm and to draw MSTs based on 57 TALE patterns obtained for X. oryzae pv. oryzicola, BAI23, and $X$. campestris pv. leersiae strains.

T3E screening. Two T3E genes, $x o p W$ and $x o p A J$, were amplified by PCR from the entire collection of strains, as previously described (16). Additionally, six strains (MAI18, MAI23, BAI5, BAI11, BAI15, and BAI23) were characterized for their entire T3E repertoire (43 selected T3E genes). Both PCR and dot-blot

TABLE 1. (Continued from previous page)

\begin{tabular}{|c|c|c|c|c|c|c|c|c|c|c|c|c|c|}
\hline Strain & Other names ${ }^{v}$ & Origin & Region & Site & Year & Host & xopAJw & xopW ${ }^{x}$ & gyrB $^{y}$ & MLSA & Vir. $^{z}$ & TAL & Reference \\
\hline MAI 24 & & Mali & O. Niger & Kogoni & 2009 & O. barthii & - & $>$ & na & - & 3 & 14 & This study \\
\hline MAI 25 & LMG26400 & Mali & O. Niger & Kogoni & 2009 & O. longistaminata & - & $>$ & II & $\mathrm{b}$ & 3 & 13 & This study \\
\hline MAI 26 & & Mali & O. Niger & Macina & 2009 & O. sativa & - & + & na & - & 1 & 1 & This study \\
\hline MAI 28 & & Mali & O. Niger & Macina & 2009 & O. sativa & + & + & na & - & 2 & 1 & This study \\
\hline MAI 29 & LMG27223 & Mali & O. Niger & Macina & 2009 & O. sativa & - & + & I & $\mathrm{c}$ & 4 & 9 & This study \\
\hline MAI 30 & & Mali & O. Niger & Macina & 2009 & O. sativa & - & + & na & - & 3 & 15 & This study \\
\hline MAI 31 & & Mali & O. Niger & Macina & 2009 & O. sativa & - & + & na & - & 4 & 15 & This study \\
\hline MAI 32 & & Mali & O. Niger & Macina & 2009 & O. sativa & - & + & na & - & 3 & 15 & This study \\
\hline MAI 33 & & Mali & O. Niger & Macina & 2009 & O. sativa & - & + & na & - & 4 & 15 & This study \\
\hline MAI 34 & & Mali & O. Niger & Macina & 2009 & O. sativa & + & + & na & - & 3 & 1 & This study \\
\hline MAI 35 & & Mali & O. Niger & Macina & 2009 & O. sativa & + & + & na & - & 2 & 1 & This study \\
\hline MAI 36 & LMG26401 & Mali & O. Niger & Ndebougou & 2009 & O. barthii & + & + & I & $\mathrm{f}$ & 2 & 11 & This study \\
\hline MAI 37 & & Mali & O. Niger & Niono & 2009 & O. sativa & - & + & na & - & 3 & 13 & This study \\
\hline MAI 38 & & Mali & Koulikoro & Dialakoroba & 2009 & O. sativa & + & + & na & - & 1 & na & This study \\
\hline MAI 39 & & Mali & Sikasso & Sélingué & 2009 & O. sativa & - & + & na & - & 0 & na & This study \\
\hline MAI 40 & & Mali & Sikasso & Sélingué & 2009 & O. sativa & + & + & na & - & 1 & 10 & This study \\
\hline MAI 41 & & Mali & Sikasso & Sélingué & 2009 & O. sativa & - & + & na & - & 1 & na & This study \\
\hline MAI 42 & LMG26402 & Mali & Sikasso & Sélingué & 2009 & O. sativa & + & + & I & $\mathrm{c}$ & 2 & 10 & This study \\
\hline MAI 43 & & Mali & Sikasso & Sélingué & 2009 & O. sativa & + & + & na & - & 3 & 10 & This study \\
\hline MAI 44 & & Mali & Sikasso & Sélingué & 2009 & O. sativa & + & + & na & - & 4 & 10 & This study \\
\hline MAI 45 & & Mali & Sikasso & Sikasso & 2009 & O. sativa & - & + & na & - & 5 & 4 & This study \\
\hline MAI 46 & LMG27224 & Mali & Koulikoro & Baguineda & 2009 & O. sativa & + & + & I & $\mathrm{g}$ & 4 & 5 & This study \\
\hline \multicolumn{14}{|c|}{ Xanthomonas sp. } \\
\hline $89 \mathrm{M}$ & & Mali & O. Niger & Molodo & 2009 & O. sativa & - & - & new slc & na & 0 & na & This study \\
\hline $97 \mathrm{M}$ & CFBP7942 & Mali & O. Niger & Ndebougou & 2009 & O. sativa & - & - & new slc & na & 0 & na & This study \\
\hline $106 \mathrm{M}$ & CFBP7943 & Mali & O. Niger & Niono & 2009 & O. sativa & - & - & new slc & na & 0 & na & This study \\
\hline $136 \mathrm{M}$ & & Mali & Sikasso & Sikasso & 2009 & O. sativa & - & - & slc7 & na & 0 & na & This study \\
\hline $120 \mathrm{M}$ & & Mali & Koulikoro & Dialakoroba & 2009 & O. sativa & - & - & $\begin{array}{l}\text { clade } X \\
\text { fuscans }\end{array}$ & na & 0 & na & This study \\
\hline \multicolumn{14}{|c|}{$\begin{array}{l}\text { X. oryzae pv. oryzicola } \\
\text { (used as controls) }\end{array}$} \\
\hline MAI 3 & $\begin{array}{l}\text { LMG27221, } \\
\text { CFBP7326 }\end{array}$ & Mali & O. Niger & Niono & 2003 & O. sativa & + & + & I & na & 5 & 3 & 13 \\
\hline MAI 8 & $\begin{array}{l}\text { LMG25979, } \\
\text { CFBP7330 }\end{array}$ & Mali & O. Niger & Niono & 2003 & O. sativa & - & $>$ & II & $\mathrm{b}$ & na & na & 13 \\
\hline MAI 10 & $\begin{array}{l}\text { LMG27222, } \\
\text { CFBP7331 }\end{array}$ & Mali & O. Niger & Niono & 2003 & O. sativa & - & $>$ & II & $\mathrm{b}$ & na & 14 & 13 \\
\hline MAI 11 & $\begin{array}{l}\text { LMG25980, } \\
\text { CFBP7335 }\end{array}$ & Mali & O. Niger & Niono & 2003 & O. sativa & - & $>$ & II & $\mathrm{b}$ & na & na & 13 \\
\hline BLS256 & $\begin{array}{l}\text { LMG27109, } \\
\text { CFBP7109 }\end{array}$ & Philippines & Los Banos & IRRI & 1985 & O. sativa & + & + & I & $\mathrm{j}$ & 5 & 17 & 6 \\
\hline LMG797 & CFBP2286 & Malaysia & na & na & 1964 & O. sativa & + & + & I & $\mathrm{j}$ & na & na & 13 \\
\hline Xoc China & & China & na & na & na & na & + & + & I & $\mathrm{c}$ & na & na & 22 \\
\hline \multicolumn{14}{|c|}{ X. campestris pv. leersiae } \\
\hline NCPPB4346 & & China & na & na & na & Leersia hexandra & - & - & $\mathrm{Xcl}$ & $\mathrm{Xcl}$ & 1 & 18 & 33 \\
\hline BAI 23 & $\begin{array}{l}\text { LMG25978, } \\
\text { CFBP7344 }\end{array}$ & Burkina & Cascades & Karfiguela & 2009 & Weeds & - & - & $\mathrm{Xcl}$ & $\mathrm{Xcl}$ & 1 & 16 & This study \\
\hline \multicolumn{14}{|c|}{$X$. oryzae pv. oryzae } \\
\hline MAI1 & $\begin{array}{l}\text { CFBP7325, } \\
\text { LMG25985 }\end{array}$ & Mali & O. Niger & Niono & 2003 & O. sativa & na & na & Xoo Africa & Xoo Africa & na & na & 13 \\
\hline BAI3 & $\begin{array}{l}\text { CFBP7321, } \\
\text { LMG25983 }\end{array}$ & Burkina & East center & Bagre & 2003 & O. sativa & na & na & Xoo Africa & Xoo Africa & na & na & 13 \\
\hline LMG12464 & CFBP1947 & Cameroon & na & na & 1980 & O. sativa & na & na & Xoo Africa & Xoo Africa & na & na & 37 \\
\hline LMG6518 & & Mali & na & na & 1985 & O. sativa & na & na & Xoo Africa & Xoo Africa & na & na & na \\
\hline PXO99 & LMG26970 & Philippines & na & na & & O. sativa & na & na & Xoo Asia & Xoo Asia & na & na & 38 \\
\hline MAFF311018 & & Japan & na & na & & O. sativa & na & na & Xoo Asia & Xoo Asia & na & na & 29 \\
\hline KACC10331 & & Korea & na & na & & O. sativa & na & na & Xoo Asia & Xoo Asia & na & na & 24 \\
\hline LMG5047 & $\begin{array}{l}\text { ATCC } 35933 \\
\text { CFBP2532 }\end{array}$ & India & na & na & 1965 & O. sativa & na & na & Xoo Asia & Xoo Asia & na & na & 42 \\
\hline \multicolumn{14}{|l|}{$X$. oryzae } \\
\hline LMG12463 & $\mathrm{X} 1-8$ & $\begin{array}{l}\text { United } \\
\text { States }\end{array}$ & Texas & na & 1987 & O. sativa & na & na & Xo US & Xo US & na & $\begin{array}{c}\text { no } \\
\text { TAL }\end{array}$ & 20 \\
\hline
\end{tabular}


hybridization methods were used to screen for presence and absence of $43 \mathrm{~T} 3 \mathrm{E}$ genes according to the methods described by Hajri et al. (16,17). The T3E gene nomenclature is as described at the Xanthomonas resource website (http://www.xanthomonas.org/).

The amplified DNA fragment corresponding to $x o p W$ was purified for selected $X$. oryzae pv. oryzicola strains using the QIAquick PCR Extraction Kit (Qiagen, Inc., Valencia, CA), and both strands were sequenced at the Proteomics and Metabolomics Facility, Colorado State University. Sequences were compared with those of Asian X. oryzae pv. oryzae and X. oryzae pv. oryzicola strains in GenBank using the BLAST program available at the NCBI (http://www.ncbi.nlm.nih.gov/BLAST/). Sequences were aligned using ClustalW. Phylogenetic trees were constructed from the aligned sequences using MEGA 5 as described above, with an NJ method and 1,000 bootstrap replicates.

\section{RESULTS}

Identification of $X$. oryzae pv. oryzicola strains. Symptomatic leaves were collected from rice fields in three and five riceproducing regions in Mali and Burkina Faso, respectively (Fig. 1). Seventy-three xanthomonad-like isolates (yellow mucoid colonies) were isolated and further characterized (Table 1). The multiplexPCR developed for the identification of $X$. oryzae pathovars was used to confirm the identity of all $X$. oryzae isolates. $X$. oryzae pv. oryzicola strain BLS256 and X. oryzae pv. oryzae strain PXO99 ${ }^{\mathrm{A}}$ were used as controls. The three expected DNA fragments (331, 691, and $945 \mathrm{bp}$ ) corresponding to X. oryzae pv. oryzicola were obtained from all but seven strains (BAI23, M89, M97, M106, M120, M136, and the X. campestris pv. leersiae strain NCPPB4346) (data not shown). No amplicons were observed for negative controls (distilled water). Amplifications for positive control DNA (PXO99 ${ }^{\mathrm{A}}$ and BLS256) were as previously described (23).

Pathogenicity analysis. All isolates were tested for pathogenicity on susceptible Nipponbare using $X$. oryzae pv. oryzicola strain BLS256 as reference. Differences in virulence were observed among the African $X$. oryzae pv. oryzicola strains. The average size of the lesions varied greatly among the isolates independently of their geographical origin or hosts (Fig. 2A and B). A comparison of means by each pair student $t$ test revealed a significant effect of $X$. oryzae pv. oryzicola isolates $(P<0.05)$. Five virulence classes were defined according to the lesion length (L) induced by $X$. oryzae pv. oryzicola, with $0=$ no symptom, $1=$ $1<\mathrm{L} \leq 10 \mathrm{~mm}, 2=10<\mathrm{L} \leq 20 \mathrm{~mm}, 3=20<\mathrm{L} \leq 30 \mathrm{~mm}, 4=30$ $<\mathrm{L} \leq 40 \mathrm{~mm}$, and $5=\mathrm{L}>40 \mathrm{~mm}$ (Fig. 2B). Of the 67 isolates obtained from Oryza spp., 7 (BAI38, MAI39, M89, M97, M106, M120, and M136) did not induce any lesion upon leaf infiltration (class 0) (Table 1; Fig. 2A). Strains BAI13, BAI17, BAI20, BAI23, BAI62, and BAI65 isolated from weeds induced lesions of variable size on rice, whereas strain BAI 23 induced only small lesions, similar to what was observed for $X$. campestris pv. leersiae strain NCPPB4346 (Table 1). Three X. oryzae pv. oryzicola strains from Burkina Faso (BAI6, BAI11, and BAI19) and one from Mali (MAI45) were particularly virulent, provoking lesions $>40 \mathrm{~mm}$ (virulence class 5).

All 67 X. oryzae pv. oryzicola strains isolated in this study and confirmed by multiplex PCR were then tested on Kitaake and Kitaake-Rxol rice, with strain BLS256 used as control (Fig. 2A and C). All the strains except BAI38 and MAI39 induced a watersoaking reaction on Kitaake $72 \mathrm{~h}$ after infiltration (data not shown). BAI38 and MAI39 did not induce any lesion upon leaf infiltration on any of the three rice varieties tested. On KitaakeRxol, the reaction was variable, with 22 strains (14 and 8 strains

TABLE 2. Primers used in the multilocus sequence analysis study for amplification of the target gene fragments

\begin{tabular}{|c|c|c|c|}
\hline Gene & Sequence forward $5^{\prime}-3^{\prime}$ & Sequence reverse $5^{\prime}-3^{\prime}$ & References \\
\hline $\operatorname{atp} D$ & GGGCAAGATCGTTCAGAT & GCTCTTGGTCGAGGTGAT & 8 \\
\hline dnaK & GGTGGAAGACCTGGTCAAGA & TCCTTGACYTCGGTGAACTC & 47 \\
\hline efP & TCATCACCGAGACCGAATA & TCCTGGTTGACGAACAGC & 8 \\
\hline lepA & AAGCSCAGGTGCTCGACTCCAAC & CGTTCCTGCACGATTTCCATGTG & 4 \\
\hline$r p o D$ & TGGAACAGGGCTATCTGACC & CATTCYAGGTTGGTCTGRTT & 47 \\
\hline
\end{tabular}

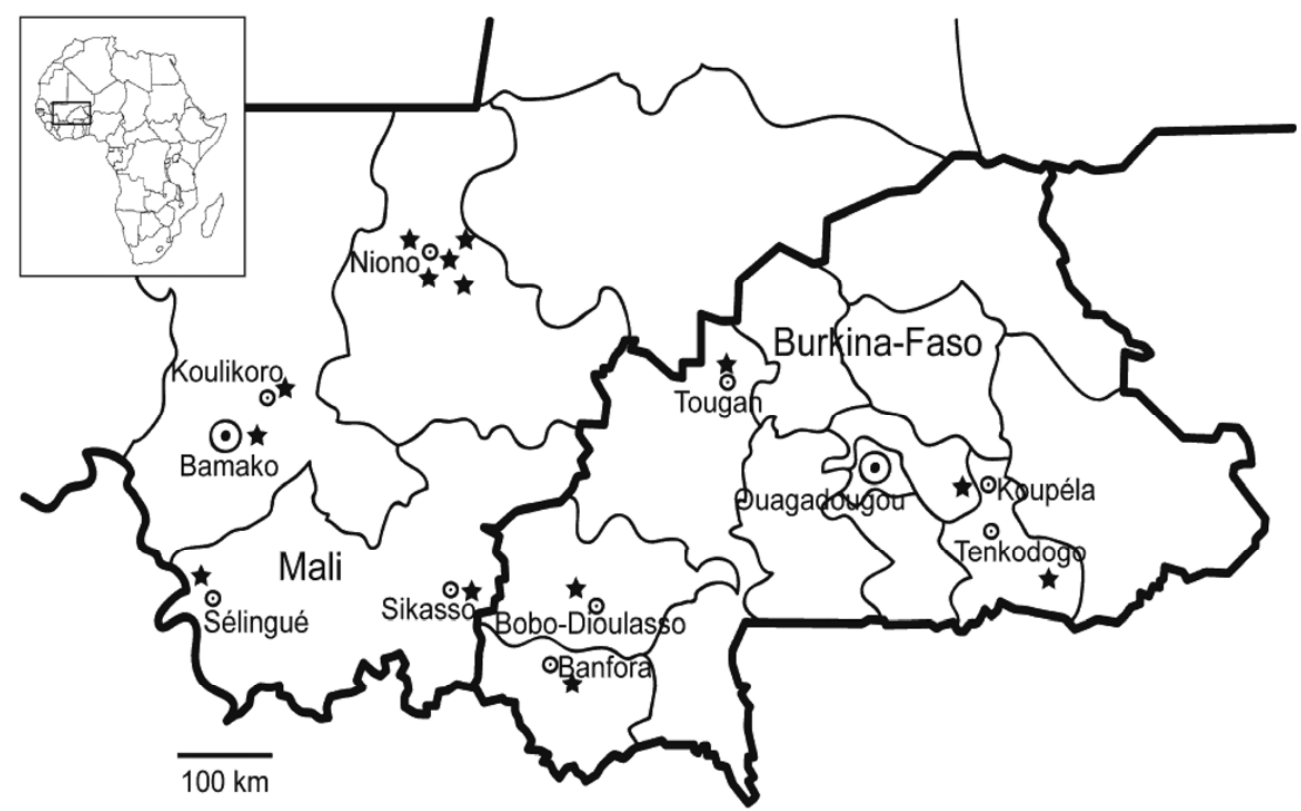

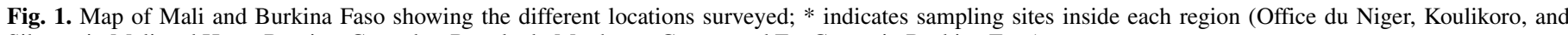
Sikasso in Mali and Hauts Bassins, Cascades, Boucle du Mouhoun, Center, and Est Center in Burkina Faso). 
from Mali and Burkina Faso, respectively) inducing HR $72 \mathrm{~h}$ after infiltration, similar to that observed with $X$. oryzae pv. oryzicola strain BLS256 (Fig. 2A and C).

MLSA. A subset of $44 X$. oryzae pv. oryzicola strains, $X$. campestris pv. leersiae strain NCPPB4346, and $9 X$. oryzae pv. oryzae strains were selected for MLSA, in which six housekeeping genes ( $a t p D, d n a K, e f p, g y r B$, lepA, and $r p o D$ ) were included. The sizes of the six partial gene sequences are $747 \mathrm{bp}(a t p D), 891 \mathrm{bp}$ (dnaK), 387 bp (efp), 528 bp (gyrB), 390 bp (lepA), and 870 bp (rpoD), giving a total of $3,813 \mathrm{bp}$ for the concatenated data set.

Among the analyzed strains, the number of haplotypes at each locus ranged from 5 (efp) to 12 (atpD). The number of polymorphic nucleotide sites varied from 7 for the least polymorphic locus (efp) to 29 for the most polymorphic locus ( $g y r B$ and $d n a K$ ) (Supplemental Table 1). Individual gene trees, as well as the phylogenetic tree based on the concatenated sequences, revealed that $X$. oryzae strains can be separated into different groups representing $X$. oryzae pv. oryzicola, $X$. oryzae pv. oryzae (Asian and African), $X$. oryzae US, and $X$. campestris pv. leersiae (Figs. 3 and 4; Supplemental Figure 1). gyrB is the most diverse gene among the six housekeeping genes included in this study and is well-suited to determine the phylogenetic structure of Xanthomonas spp. (33). The phylogenetic tree based on partial gyrB sequences revealed seven groups among the $X$. oryzae and $X$. campestris pv. leersiae strains (Table 1; Fig. 3). Within pv. oryzae, subgroups of strains corresponded to their geographic origin ( $X$. oryzae pv. oryzae Africa and Asia and $X$. oryzae US), confirming the results of previous studies $(13,17,46)$. Three subgroups were identified among the $43 X$. oryzae pv. oryzicola strains (Fig. 3). gyrB subgroup I included $X$. oryzae pv. oryzicola strains from Africa and Asia whereas the other two subgroups (II and III) only contained African X. oryzae pv. oryzicola strains. Interestingly, subgroup III comprises strains that originated only from Bagre, Burkina Faso (Table 1). This subgroup also formed a separate clade in the consensus split network (Fig. 4, clade a).

Based on all partial gene sequences analyzed, the $X$. oryzae pv. oryzicola strain from China (X. oryzae pv. oryzicola China) grouped together with African strains (Fig. 3, subgroup I; and Fig. 4 , clade c). In some cases, other housekeeping genes such as atpD, lepA, and efp may be more informative than $g y r B$ to distinguish strains within $X$. oryzae pv. oryzicola. For example, gyr $B$ sequences of $X$. oryzae pv. oryzicola strains LMG797 and BLS256 are identical and grouped together with African $X$. oryzae pv. oryzicola strains (Fig. 3, subgroup I) but they formed a separate clade in analyses based on $a t p D, \operatorname{lep} A$, and efp. In addition to gyr $B$ analysis, we used the concatenated partial sequences of all six housekeeping genes to construct a phylogenetic tree and a consensus split network; this analysis supported the division of

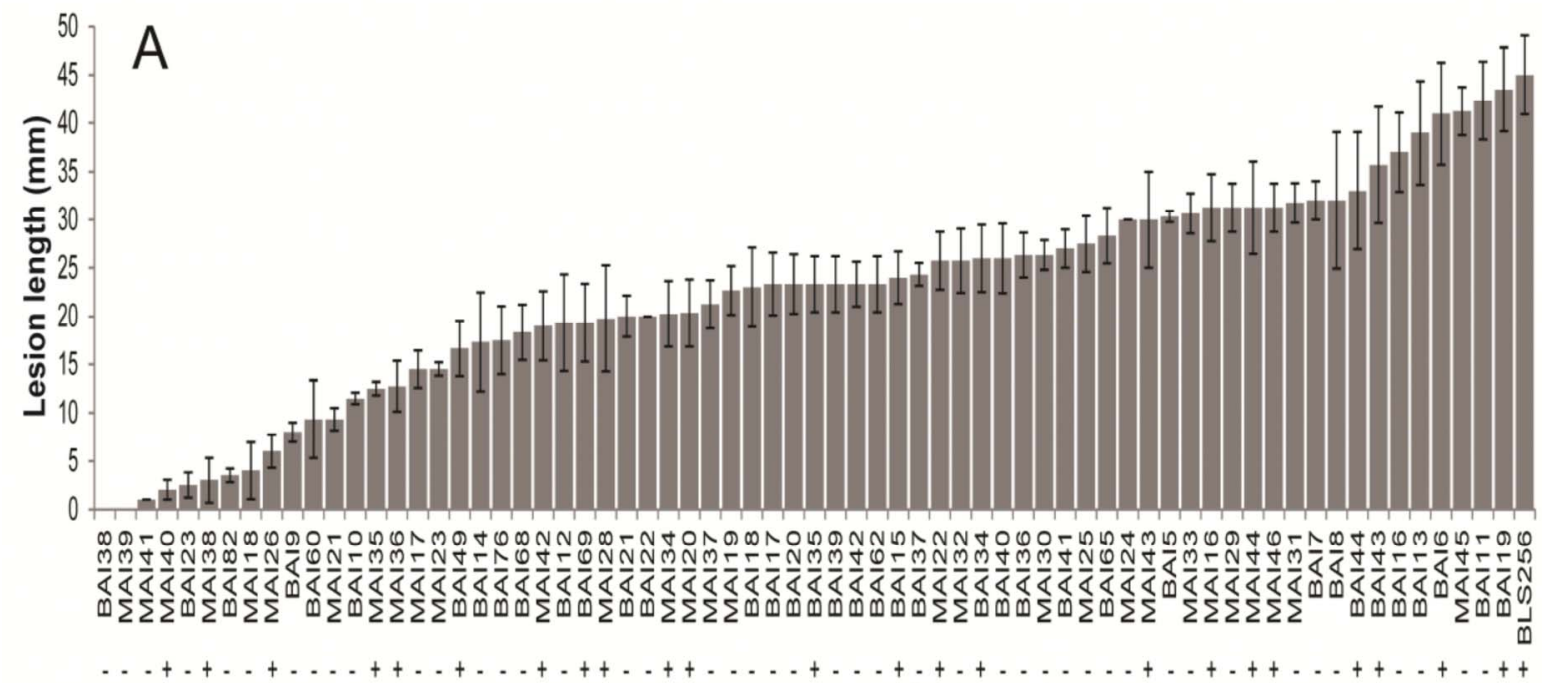

$X$. oryzae pv. oryzicola strains
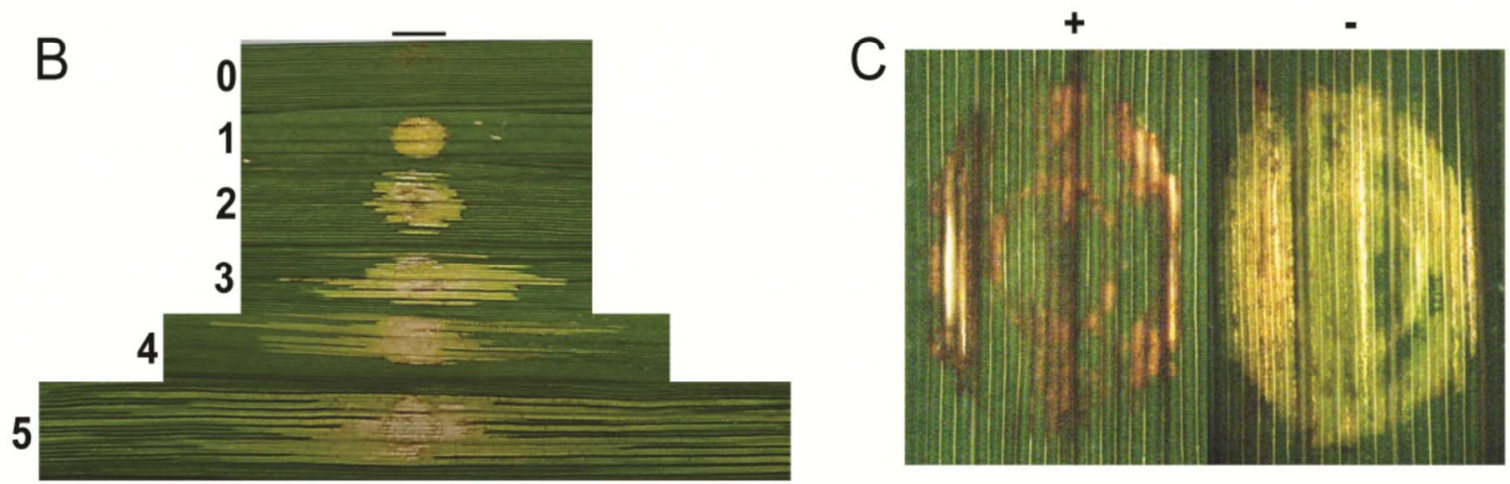

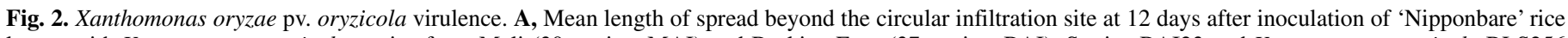

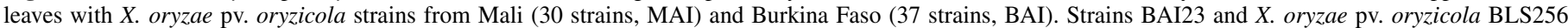

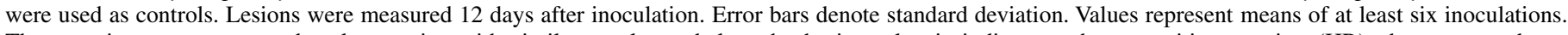

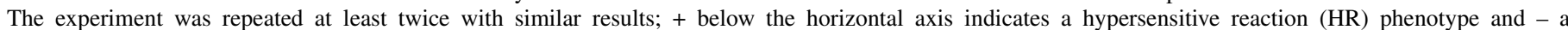

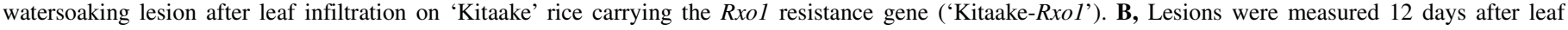

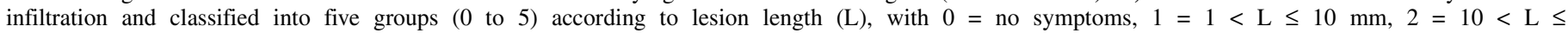

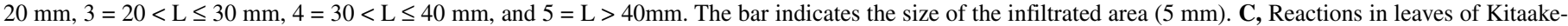

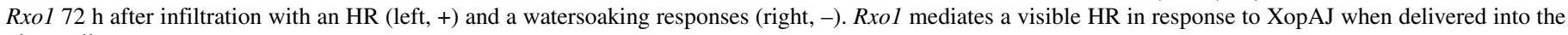
plant cell. 


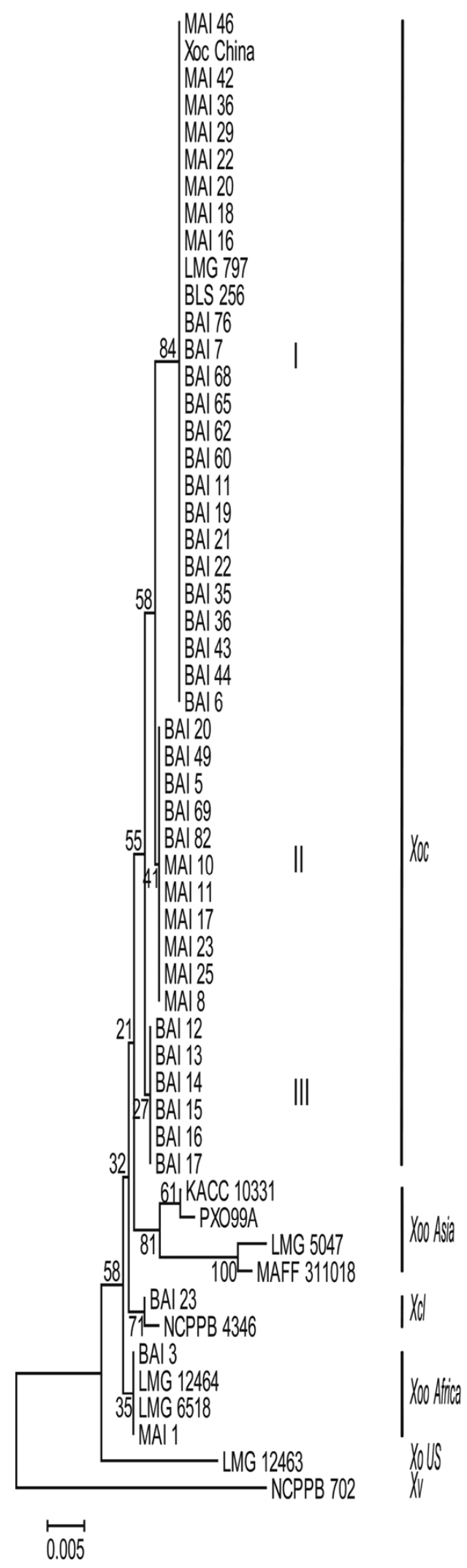

Fig. 3. Phylogenetic tree based on partial gyrB sequences from strains of Xanthomonas oryzae pv. oryzae (Xoo), X. campestris pv. leersia $(X c l)$, and $X$. oryzae pv. oryzicola $(\mathrm{Xoc})$. The tree was constructed with Mega 5 software using a neighbor-joining algorithm with 1,000 bootstrap replicates and rooted with the $X$. vasicola pv. vasculorum strain NCPPB702 (Xv). Bootstrap support of the different groups is represented on the tree at the different nodes. The branch length is proportional to divergence and the 0.005 scale represents $0.5 \%$ change.
African and Asian $X$. oryzae pv. oryzicola strains into separate subgroups, as observed with gyrB-based analysis (Fig. 4; Supplemental Figure 2). In this analysis, $X$. oryzae pv. oryzicola strains can be divided into 10 subgroups (a to j) (Fig. 4; Table 1). Interestingly, four clades (a, e, h, and i) were composed only of strains from Burkina Faso (Fig. 4). Other clades (b, c, f, and g) were composed of strains from Mali and Burkina Faso, with the Chinese strain of $X$. oryzae pv. oryzicola also found in clade c (Fig. 4). The two other Asian $X$. oryzae pv. oryzicola strains formed one clade $(\mathrm{j})$, and can be separated from all African $X$. oryzae pv. oryzicola strains. Although $X$. oryzae pv. oryzicola strains isolated from wild rice and weeds were scattered all over the different clades, one clade (f) was composed solely of strains isolated from wild rice (Fig. 4; Table 1). The protein analysis of the concatenated partial sequences of all six housekeeping genes confirmed the overall picture within the $X$. oryzae species (i.e., the differentiation into pathovars and the different $X$. oryzae pv. oryzicola groups). Although the distinction between $X$. oryzae pv. oryzicola groups I and II is less clear, group III is clearly differentiated at the amino acid level.

$X$. campestris pv. leersiae strain NCPPB4346 was contained within the $X$. oryzae cluster independently of the gene analyzed (Figs. 3 and 4). Strain NCPPB4346 isolated from Leersia hexandra in China showed relatively high sequence relatedness with both $X$. oryzae pathovars, although it could not be assigned to either pathovar (Figs. 3 and 4). Strain BAI23 (isolated on weeds) showed high sequence similarity with $X$. campestris pv. leersiae strain NCPPB4346 for all genes analyzed (Fig. 3; data not shown). This cluster (named $X$. campestris pv. leersiae), comprising two strains (BAI23 and NCPPB4346), could be differentiated from the other described lineages $(X$. oryzae pv. oryzae Africa and Asia, $X$. oryzae US, and $X$. oryzae pv. oryzicola Africa and Asia) within $X$. oryzae, and was supported by a high bootstrap value in the $\operatorname{gyr} B$ phylogenetic tree (Fig. 3). Pathogenicity of these strains and identification of additional strains from $L$. hexandra should be performed to examine the position of these strains within $X$. oryzae and for the assignment of a possible new $X$. oryzae pathovar. Similar reflections can be made for $X$. oryzae US.

Analysis of the TALE repertoire. TALEs presumably contribute in a significant manner to $X$. oryzae pv. oryzicola virulence, as notably suggested by the over-representation of this family of T3Es within $X$. oryzae pv. oryzicola genomes. Because $T A L E$ genes are potentially highly subjected to duplication and recombination events, assessing the diversity of TALE repertoires is often used as a marker to estimate genetic distances in a set of strains. To that end, we evaluated the diversity of the TALE repertoire of $55 X$. oryzae pv. oryzicola and $2 X$. campestris pv. leersiae strains (BAI23 and NCPPB4346) upon Southern-blot analysis. In total, 18 haplotypes were identified, including 16 among $X$. oryzae pv. oryzicola strains and 2 among strains of $X$. campestris pv. leersiae. The number of bands ranged from 4 (X. campestris pv. leersiae) to 15 (X. oryzae pv. oryzicola) (Fig. 5; Table 1). MST analysis shows that three haplotypes are shared between strains from Mali and Burkina Faso, whereas eight and four are specific to Burkina Faso and Mali, respectively. The distribution of the haplotypes shows a low degree of geographic correlation (Table 1; Fig. 6). Strains MAI3 and MAI10 (isolated in 2003 in Mali) showed TALE patterns identical to those of $X$. oryzae pv. oryzicola strains isolated 7 to 8 years later in Burkina Faso and Mali (Table 1; Fig. 6). Although not identical, the TALE patterns of $X$. campestris pv. leersiae strains NCPPB4346 and BAI23 were very similar, with 4 to 5 bands, respectively (data not shown). X. campestris pv. leersiae strains have two loci in common and are more distant from $X$. oryzae pv. oryzicola haplotypes. The dendrogram generated based on TALE pattern showed that $X$. campestris pv. leersiae strains are closely related by high bootstrap value $(86 \%)$ (data not shown). 
T3E screening. PCR was used to screen the entire $X$. oryzae pv. oryzicola collection for presence or absence of two T3E genes, $x o p W$ and $x o p A J$. The $x o p W$ gene was amplified in all $X$. oryzae pv. oryzicola strains with two different sizes of amplicon, depending on the strain (Table 1; amplicons are indicated as + for $600 \mathrm{bp}$ and $>$ for 1,650 bp). The 1,650-bp fragment corresponds to the insertion of an IS1112 element of 1,050 bp in xopW. No amplicon was observed from $X$. campestris pv. leersiae strains BAI23 and NCPPB4643 and strains M89, M97, M106, M120, and M136 (Table 1). The 1,650-bp fragment is amplified in $13 \mathrm{X}$. oryzae pv. oryzicola strains, all originating from Mali and Burkina Faso (Table 1). Among these, 11 belong to the gyrB group II and MLSA group b (Table 1). The other two strains (MAI21 and MAI24) were not included in the MLSA analysis.

$X o p W$ sequences were then determined for a subset of strains (MAI3, MAI10, MAI11, MAI18, MAI20, MAI22, BAI7, BAI15, BAI17, BAI20, BAI21, BAI22, X. oryzae pv. oryzicola China, BLS256, and LMG797). Nucleotide sequence analysis (analyzed without the IS element) showed that African $X$. oryzae pv. oryzicola strains differ by four single-nucleotide polymorphisms (SNP) at positions 8, 73, 330, and 362 (Table 3). The phylogenetic tree based on $x$ op $W$ sequences revealed four groups among the $X$. oryzae pv. oryzicola strains, with African $X$. oryzae pv. oryzicola more closely related to the Chinese strain than to Malaysian and Philippine strains (data not shown).

Although XopAJ is detected in all Asian X. oryzae pv. oryzicola, its presence is highly variable among African $X$. oryzae pv. oryzicola and depends on the strain's origin and year of collection (Table 1; Fig. 7). In general, African $X$. oryzae pv. oryzicola strains that are positive for $x o p A J$ belong to the $g y r B$ group I (Fig. $2 \mathrm{~A}$; Table 1). Interestingly, all $X$. oryzae pv. oryzicola strains from Bagre, Burkina Faso, (group gyrB III and MLSA a) are negative for xopAJ (Table 1). Function of xopAJ was confirmed by leaf infiltration on Kitaake-Rxol rice (Fig. 2A and C). All the $X$. oryzae pv. oryzicola strains that were PCR positive for xopAJ

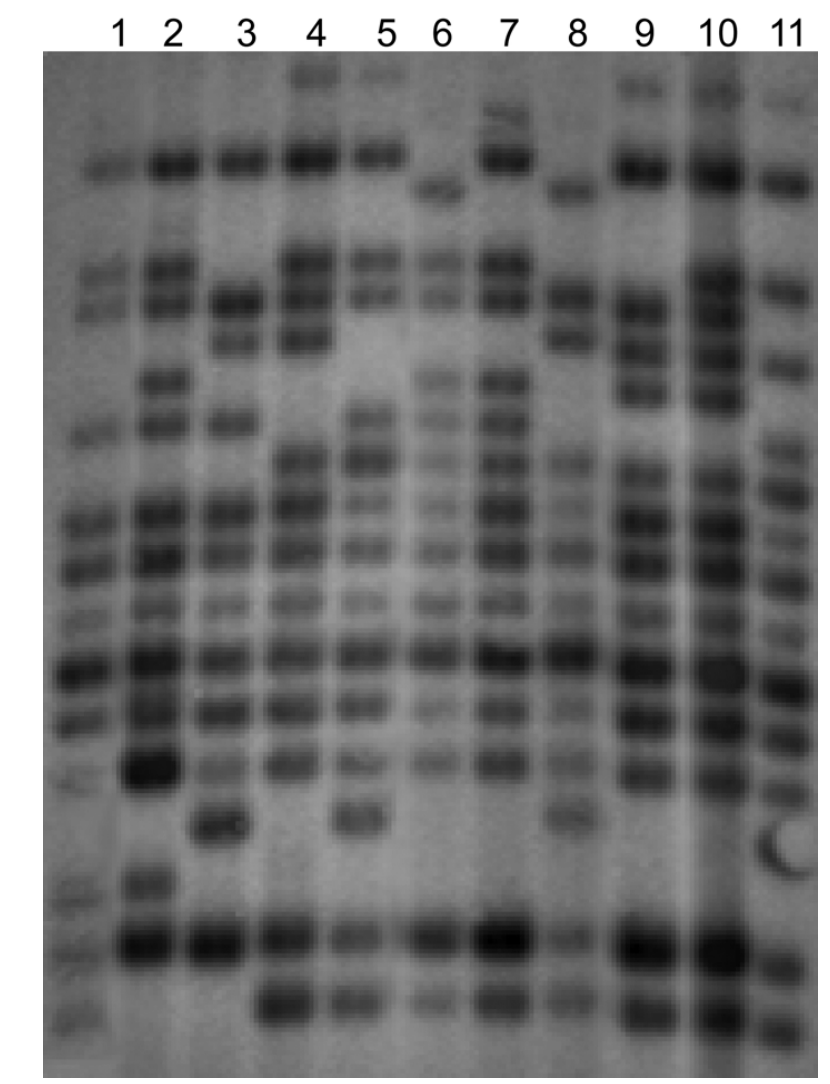

Fig. 5. Hybridization patterns or haplotypes (Hts) obtained with $a v r X a 7$ as a probe on BamHI-digested genomic DNA obtained from the following Xanthomonas oryzae pv. oryzicola strains: lane 1, BAI41 (Ht8); lane 2, BLS256 (Ht17); lane 3, MAI3 (Ht3); lane 4, MAI45 (Ht4); lane 5, MAI36 (Ht11); lane 6, MAI31 (Ht15); lane 7, MAI29 (Ht9); lane 8, MAI28 (Ht1); lane 9, MAI25 (Ht13); lane 10, MAI23 (Ht14); and lane 11, MAI22 (Ht6).

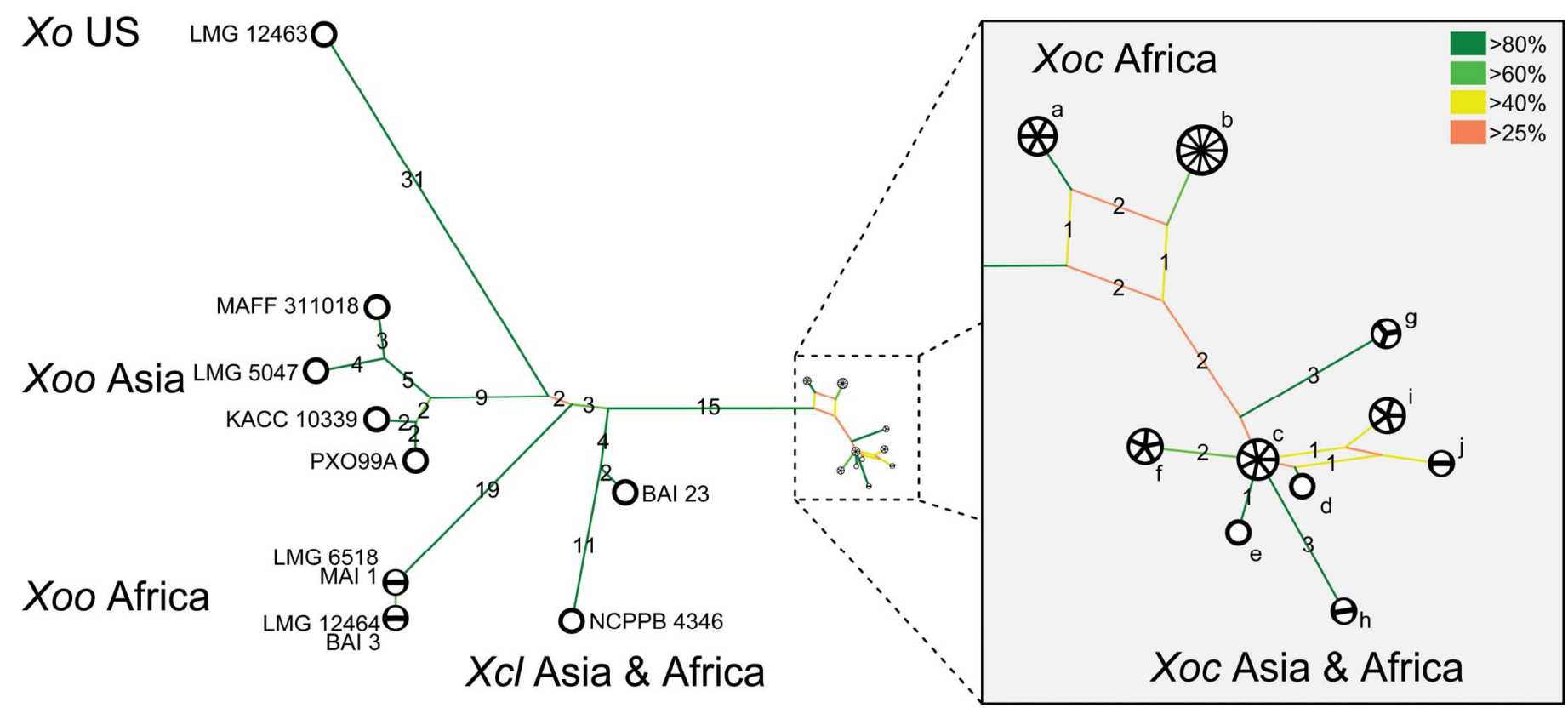

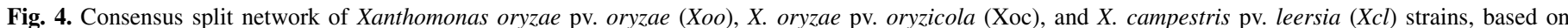

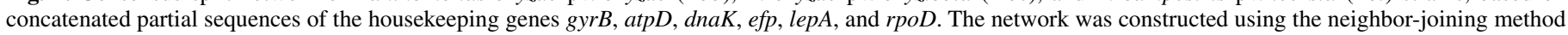

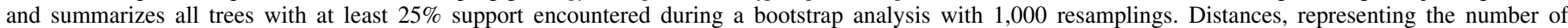

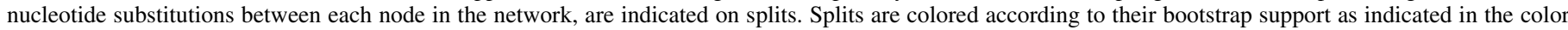

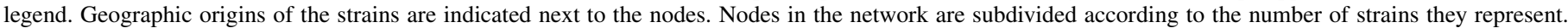

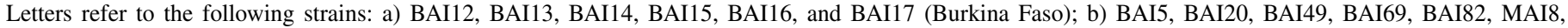

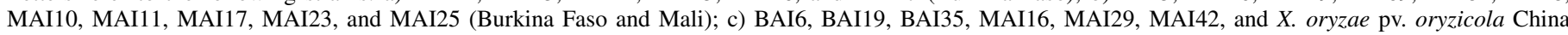

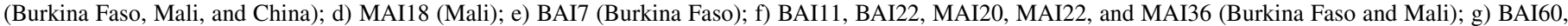

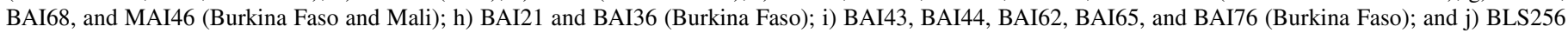
and LMG797 (Philippines and Malaysia). 
induced an HR 72 hai whereas the strains that were PCR negative for $x o p A J$ induced watersoaking lesions on Kitaake-Rxol (Fig. 2A). In Mali, the percentage of $X$. oryzae pv. oryzicola strains harboring a functional $x o p A J$ gene varied between years (Table 1).

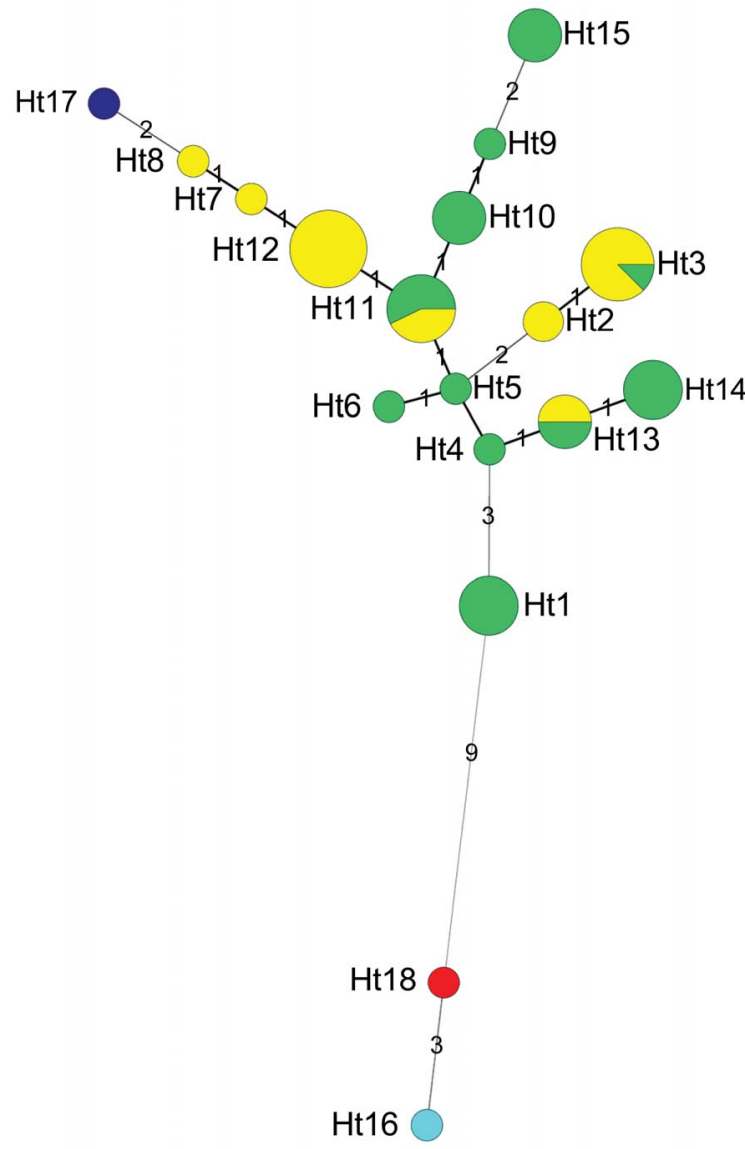

Fig. 6. Minimum spanning tree of Xanthomonas oryzae pv. oryzicola haplotypes (Hts) from Mali and Burkina Faso, BLS256, and X. campestris pv. leersia NCPP4346 and BAI23. Each Ht is represented by a node, the size of which is proportional to the number of strains that it comprises. Bicolor pie charts indicate Hts of X. oryzae pv. oryzicola strains from Mali (green) and Burkina Faso (yellow). X. oryzae pv. oryzicola haplotypes corresponding to BLS256 and X. campestris pv. leersiae strains NCPP4346 and BAI23 are depicted in dark blue, red, and light blue, respectively. Locus differences are represented by the value positioned in between the nodes.
The entire repertoire of T3E was determined by PCR and blot analysis of 43 known Xanthomonas effectors in six strains (MAI18, MAI23, BAI5, BAI11, BAI15, and BAI23) belonging to five different MLSA groups $(\mathrm{a}, \mathrm{b}, \mathrm{d}, \mathrm{f}$, and $X$. campestris $\mathrm{pv}$. leersiae) (Table 1). Data were compared with previous work by Hajri et al. (17). Strains MAI18, BAI11, and BAI15 have identical repertoires of $24 \mathrm{~T} 3 \mathrm{E}$ genes (Fig. 7). The T3E repertoires of strain MAI23 and BAI5 differ slightly from this, harboring an IS insertion in xopW (MAI23) (Fig. 7; Table 1) or xopAD (BAI5) (Fig. 7). Finally, the $X$. campestris pv. leersiae strain BAI23 differed from $X$. oryzae pv. oryzicola strains in that it lacks xopO and $x o p W$ (Fig. 7; Table 1).

\section{DISCUSSION}

Surveys conducted in 2009, 2010, and 2011 in major riceproducing areas of Mali and Burkina Faso revealed the presence of BLS disease in most rice fields (49). These areas correspond to diverse agroecological regions in Mali and Burkina Faso where BLS is considered an emerging disease $(13,49)$. Although BLS disease was reported in Africa and Madagascar in the 1980s (5), no breeding strategy was developed for controlling the disease, nor have effective resistance sources been identified in African rice germplasm. This lack of resistant germplasm has created a favorable environment for the disease to spread. In this study, we assembled a comprehensive collection of $67 \mathrm{X}$. oryzae pv. oryzicola strains and evaluated the collection for its genetic and pathogenic diversity, yielding new insights into the $X$. oryzae pv. oryzicola population structure in West Africa.

$X$. oryzae pv. oryzicola is found on a broad range of alternate hosts in Asia, including cultivated and wild rice species, weeds, and grasses (family Poaceae) (30). Our work confirms that perennial weeds, grasses, and wild rice are alternate hosts and suggests that they may represent important reservoirs for the pathogen during intercropping seasons in West Africa. The significance of alternate hosts in the life cycle of $X$. oryzae pv. oryzicola in African rice-growing areas needs further investigation.

A high degree of phenotypic diversity was observed among African strains of $X$. oryzae pv. oryzicola. Most $X$. oryzae pv. oryzicola strains were able to cause typical BLS symptoms on rice but the severity of the symptoms varied greatly. Six virulence groups were defined upon inoculation on Nipponbare rice. No correlation was observed between virulence group, site of collection, host plant, or year of collection, suggesting that strongly and weakly virulent strains coexist in the same environments. Interestingly, two $X$. oryzae pv. oryzicola strains (BAI38 and

TABLE 3. Sequence variations for xopW gene of Xanthomonas oryzae pv. oryzicola strains used in this study

\begin{tabular}{|c|c|c|c|c|c|c|}
\hline \multirow[b]{2}{*}{ Strains } & \multirow[b]{2}{*}{ Sequence size } & \multirow[b]{2}{*}{$x o p W^{\mathrm{a}}$} & \multicolumn{4}{|c|}{ Polymorphic sites $^{b}$} \\
\hline & & & 8 & 73 & 330 & 362 \\
\hline BAI7 & 508 & + & A & A & $\mathrm{C}$ & G \\
\hline BAI15 & 508 & + & A & A & $\mathrm{C}$ & G \\
\hline BAI17 & 508 & + & A & A & $\mathrm{C}$ & G \\
\hline BAI21 & 508 & + & A & A & $\mathrm{C}$ & G \\
\hline BAI22 & 508 & + & A & A & $\mathrm{C}$ & G \\
\hline MAI3 & 508 & + & A & A & $\mathrm{C}$ & G \\
\hline MAI18 & 508 & + & A & A & $\mathrm{C}$ & $\mathrm{G}$ \\
\hline MAI20 & 508 & + & A & A & $\mathrm{C}$ & G \\
\hline MAI22 & 508 & + & A & A & $\mathrm{C}$ & $\mathrm{G}$ \\
\hline BAI20 & 573 & $>$ & A & A & $\mathrm{C}$ & G \\
\hline MAI10 & 573 & $>$ & A & A & $\mathrm{C}$ & G \\
\hline MAI11 & 573 & $>$ & A & A & $\mathrm{C}$ & G \\
\hline$X$. oryzae pv. oryzicola China & 508 & + & A & $\mathrm{C}$ & $\mathrm{C}$ & G \\
\hline BLS256 & 508 & + & G & $\mathrm{C}$ & A & A \\
\hline LMG797 & 508 & + & G & $\mathrm{C}$ & A & A \\
\hline
\end{tabular}

a Presence of $x \circ \mathrm{p} W$; > indicates that a DNA arrangement (1,050-bp insertion element) was identified within the $x o p W$ gene.

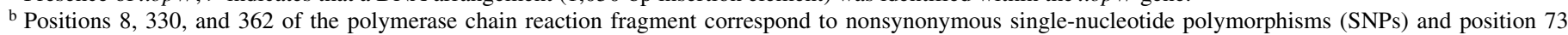
corresponds to a synonymous SNP. 
MAI39) isolated from $O$. sativa did not cause any symptoms upon leaf inoculation of rice. These strains either lack important virulence factors or possess specific effectors that induce an incompatible reaction on the varieties tested in this study, which included a very narrow range of Japonica rice varieties. Inoculation of these strains on additional rice varieties is needed to determine whether these strains are truly nonpathogenic. The characterization of their T3E and TALE repertoires may also provide clues as to why they do not induce disease symptoms.

We isolated several non- $X$. oryzae strains from rice leaves that look like Xanthomonas strains on the selective media used. These strains did not provoke any symptoms on rice. Other alternative hosts were not tested. Nonpathogenic xanthomonads that do not match any recognized Xanthomonas sp. and which occupy a unique position within the genus Xanthomonas have been reported to be commonly associated with rice $(10,11)$. Their ecological role on the rice plant or interaction with pathogenic Xanthomonas spp. needs to be further investigated. Another intriguing strain, BAI23, isolated from an unidentified weed in Burkina Faso, was not virulent on rice. $X$. campestris pv. leersiae strain NCPPB4346, a pathogen of $L$. hexandra and previously shown to group close to $X$. oryzae (32), was included in our analyses to investigate its relatedness to the Burkina Faso strain. Together, the two strains clearly form a distinct genetic cluster. They also differ from $X$. oryzae pv. oryzicola strains by the absence of three T3Es (xоpO, xорAJ, and xоpW) and a reduced number of TALE genes.

Given that strain BAI23 was isolated from a symptomatic native grass in Burkina Faso, it is very plausible that this strain may colonize Leersia plants.

In this study, multiple lines of evidence show that there are different subgroups among African $X$. oryzae pv. oryzicola strains. One group contains both African and Asian strains, suggesting that some of the African X. oryzae pv. oryzicola strains may have originated from Asia, or vice versa. Interestingly, some African strains also grouped separately from the African-Asian group. Following the recent expansion of rice cultivation, Mali and Burkina Faso have imported rice varieties from abroad, notably from Asia. X. oryzae pv. oryzicola is a seedborne and seedtransmitted pathogen (50). The importation of rice seed and movement of plant germplasm without sanitary controls would have offered an opportunity for the introduction of $X$. oryzae pv. oryzicola strains into Africa.

Our data, based on MLSA, T3E repertoire, and RLFP TALE analyses, show that the $X$. oryzae pv. oryzicola populations in Burkina Faso and Mali are highly diverse at each site surveyed. The MLSA analysis indicates that the degree of housekeeping gene sequence diversity among the African $X$. oryzae pv. oryzicola strains is even higher than that between Asian and African $X$. oryzae pv. oryzicola strains. Therefore, it is difficult to make a clear distinction among $X$. oryzae pv. oryzicola strains based on their geographical origin (as is the case for $X$. oryzae pv. oryzae strains). Based on a very limited collection of $X$. oryzae pv. oryzicola strains, Hajri et al. (17) stated that Asian X. oryzae pv. oryzicola (strains from Malaysia and the Philippines) are clearly distinguishable from African $X$. oryzae pv. oryzicola and might have evolved from African strains. Using RFLP and VNTR analysis, Raymundo et al. (35) and Zhao et al. (56) studied the diversity of an $X$. oryzae pv. oryzicola collection from the Philippines and China, respectively. They both reported a high diversity within Asian X. oryzae pv. oryzicola strains. The number of Asian $X$. oryzae pv. oryzicola strains included in our study is very limited and may not reflect the true genetic diversity among Asian $X$. oryzae pv. oryzicola. Including additional $X$. oryzae pv. oryzicola strains from Asia and other countries where the disease is reported (i.e., Madagascar) could help to clarify the origin and evolution of $X$. oryzae pv. oryzicola in Africa.

T3Es and their repertoire represent gene candidates that play a role in the adaptation of plant-pathogenic bacteria to their hosts (16). Some T3Es, such as $x o p X$ and $x o p Z$, are involved in interactions between rice and $X$. oryzae pv. oryzae. In our study, the distribution of $43 \mathrm{~T} 3 \mathrm{E}$ genes has been investigated in one $X$. campestris pv. leersiae strain and six $X$. oryzae pv. oryzicola strains belonging to different MLSA groups. All share a common repertoire of $40 \mathrm{~T} 3 \mathrm{Es}$, with only $4 \mathrm{~T} 3 \mathrm{E}$ genes (xopO, xоpW, $x o p A J$, and $x o p A D$ ) differing in the presence and absence or the presence of an IS element in the gene. These results confirm previous work by Hajri et al. (17). Our virulence data suggest that these genes are not major virulence factors and do not confer any fitness advantage or disadvantage to African $X$. oryzae pv. oryzicola. The effector xopAJ was only partially distributed among $X$. oryzae pv. oryzicola strains, despite its complete distribution among Asian $X$. oryzae pv. oryzicola. The presence or absence of the xopAJ gene among African $X$. oryzae pv. oryzicola strains is not correlated with the geographic origin or virulence of the strains or to the history of rice cultivation in the different regions. Therefore, the deployment of Rxol gene-containing varieties may not be an appropriate breeding strategy for controlling BLS disease in Africa.

TALEs of Xanthomonas spp. are important virulence factors which activate host genes that facilitate bacterial colonization and spread in the plant (7). TALE genes have expanded to many copies in the X. oryzae pv. oryzicola genome. Their function has not been studied in African $X$. oryzae pv. oryzicola, and the reason why they are so abundant in $X$. oryzae pv. oryzicola isolated on wild rice species and weeds remains unclear. Southern hybridization experiments previously revealed that African $X$. oryzae pv. oryzicola genomes contain many copies of TALE genes $(2,19,37)$, and genome assembly identified 26 TALEs in the Philippine strain $X$. oryzae pv. oryzicola BLS256 (6). We show here that strain BLS256 exhibits a specific TALE pattern different from that of African X. oryzae pv. oryzicola strains. Similar TALE haplotypes were detected in strains isolated from different locations and different phylogenetic groups in Mali and Burkina Faso. The presence of common haplotypes of $X$. oryzae pv. oryzicola in certain locations could be explained by the introduction of strains

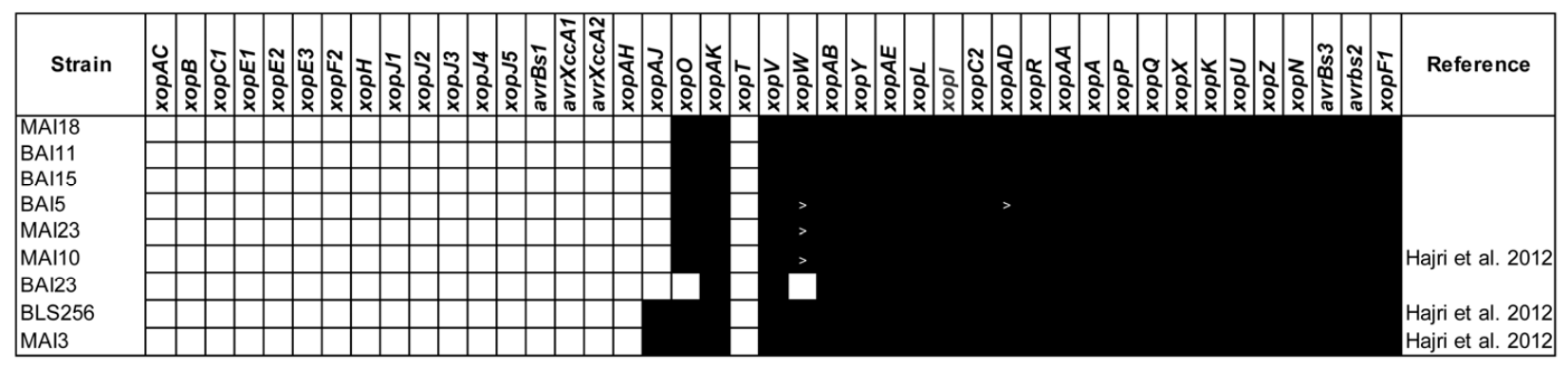

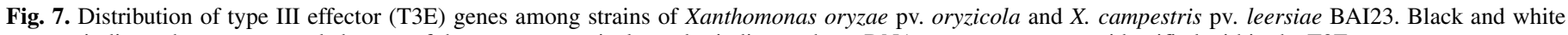
squares indicate the presence and absence of the gene, respectively, and > indicates that a DNA rearrangement was identified within the T3E gene. 
through short-distance migration from neighboring regions or through long-distance importation from rice seed from other countries. Determining the identity of the TALE in African $X$. oryzae pv. oryzicola will determine whether targets of TALE are conserved among $X$. oryzae pv. oryzicola strains from diverse origins, as seems to be the case in $X$. oryzae pv. oryzae (47). Strains of $X$. oryzae pv. oryzae of diverse geographic origins and of different genetic lineages all rely on the induction of OsSWEET14, which encodes a sugar transporter, as a major susceptibility target (37). Recent studies aiming at predicting the targets of BLS256 indicate that targeting SWEETs is not a strategy favored by $X$. oryzae pv. oryzicola (14), although introduction of $X$. oryzae pv. oryzae TALEs targeting SWEETs provided a gain of virulence to BLS256 (47).

Using AFLP markers, Escalon et al. (12) revealed the genetic diversity among specific groups of $X$. citri $p v$. citri that correlated with their geographic origin. Although AFLP markers cover the genome and are theoretically neutral, the technique is still labor intensive and time consuming. MLVA technology is currently the most advanced tool for fast, high-throughput, reliable, and highresolution intraspecific molecular typing of $X$. oryzae pv. oryzicola (56). We are now extending the MLVA analysis to our entire collection of $X$. oryzae pv. oryzicola. Also, draft genome sequences of several African $X$. oryzae pv. oryzicola strains and one $X$. campestris pv. leersiae strain are now available, and their analysis should help to explore allelic variations among T3Es and to identify other genomic factors that may play a role in virulence.

In conclusion, our study showed a high diversity within African $X$. oryzae pv. oryzicola across rice-growing areas. No correlation was found between virulence groups, sites of collection, host plants, and year of collection. Future studies are necessary on a larger collection of $X$. oryzae pv. oryzicola at a broader geographic scale to investigate pathogenic variability and population genetic structure. The virulence groups we characterized provide a basis for selecting strains useful for identifying and selecting effective sources of resistance for local rice breeding programs. Given the severity and extent of BLS epidemics in recent years in Africa, achieving stable resistance against this disease is an important goal for rice breeding programs. Due to the high virulence and high level of genetic diversity of African X. oryzae pv. oryzicola, a strategy for breeding for resistance to BLS needs to be urgently defined.

\section{ACKNOWLEDGMENTS}

I. Wonni was supported by an IRD DPF (Direction des Programmes de recherche et de la Formation au Sud) fellowship. This work was partially supported by INERA (Burkina Faso), LBMA (Mali, Bamako), and the International Foundation For Science grant awarded to I. Wonni (C/48131 and C/4813-2). Work at IRD Montpellier was supported by grants from IRD (Action incitative from the Department Environment and Resources), UMR Resistance des Plantes aux Bioagresseurs, the French Direction Générale de l'Armement (contract number 201034 006), and the French Agence Nationale de la Recherche (ANR-2010-BLAN-1723). V. Verdier (IRD) was supported by a Marie Curie IOF Fellowship (EU Grant PIOF-GA-2009-235457) while at Colorado State University (Bioagricultural Sciences and Plant Management). We thank M. Traore (IER Sikasso) and many other colleagues at IER Mali and INERA Burkina-Faso for their assistance during field surveys, J. Vandroemme (ILVO) for his help in preparing Figure 4, C. Van Malderghem (ILVO) for excellent assistance in the MLSA work, and L. Poulin for her help with data analysis.

\section{LITERATURE CITED}

1. Adhikari, T. B. 1994. Phenotypic diversity of Xanthomonas oryzae pv. oryzae in Nepal. Plant Dis. 78:68-72.

2. Adhikari, T. B., Cruz, C., Zhang, Q., Nelson, R. J., Skinner, D. Z., Mew, T. W., and Leach, J. E. 1995. Genetic diversity of Xanthomonas oryzae pv. oryzae in Asia. Appl. Environ. Microbiol. 61:966-971.
3. Adhikari, T. B., and Mew, T. W. 1985. Hostparasite relationship in bacterial leaf streak of rice caused by Xanthomonas oryzae pv. oryzicola. Nepal J. Agric. 16:134-141.

4. Almeida, N. F., Yan, S., Cai, R., Clarke, C. R., Morris, C. E., Schaad, N. W., Schuenzel, E. L., Lacy, G. H., Sun, X., Jones, J. B., Castillo, J. A., Bull, C. T., Leman, S., Guttman, D. S., Setubal, J. C., and Vinatzer, B. A. 2010. PAMDB, a multilocus sequence typing and analysis database and website for plant-associated microbes. Phytopathology 100:208-215.

5. Awoderu, V. A., Bangura, N., and John, V. T. 1991. Incidence, distribution and severity of bacterial diseases on rice in West Africa. Trop. Pest Manage. 37:113-117.

6. Bogdanove, A. J., Koebnik, R., Lu, H., Furutani, A., Angiuoli, S. V., Patil, P. B., Van Sluys, M. A., Ryan, R. P., Meyer, D. F., Han, S. W., Aparna, G., Rajaram, M., Delcher, A. L., Phillippy, A. M., Puiu, D., Schatz, M. C., Shumway, M., Sommer, D. D., Trapnell, C., Benahmed, F., Dimitrov, G., Madupu, R., Radune, D., Sullivan, S., Jha, G., Ishihara, H., Lee, S. W., Pandey, A., Sharma, V., Sriariyanun, M., Szurek, B., Vera-Cruz, C. M., Dorman, K. S., Ronald, P. C., Verdier, V., Dow, J. M., Sonti, R. V., Tsuge, S., Brendel, V. P., Rabinowicz, P. D., Leach, J. E., White, F. F., and Salzberg, S. L. 2011. Two new complete genome sequences offer insight into host and tissue specificity of plant pathogenic Xanthomonas spp. J. Bacteriol. 193:5450-5464.

7. Bogdanove, A. J., Schornack, S., and Lahaye, T. 2010. TAL effectors: finding plant genes for disease and defense. Curr. Opin. Plant Biol. 13:394-401.

8. Bui Thi Ngoc, L., Verniere, C., Jouen, E., Ah-You, N., Lefeuvre, P., Chiroleu, F., Gagnevin, L., and Pruvost, O. 2009. Amplified fragment length polymorphism and multilocus sequence analysis-based genotypic relatedness among pathogenic variants of Xanthomonas citri pv. citri and Xanthomonas campestris pv. bilvae. Int. J. Syst. Evol. Microbiol. 60:515525.

9. Chen, C., Zheng, W., Huang, X., Zhang, D., and Lin, X. Q. 2006 Major QTL conferring resistance to bacterial leaf streak. Agric. Sci. China 5:216-220.

10. Cottyn, B., Debode, J., Regalado, E., Mew, T. W., and Swings, J. 2009. Phenotypic and genetic diversity of rice seed-associated bacteria and their role in pathogenicity and biological control. J. Appl. Microbiol. 107:885897.

11. Cottyn, B., Regalado, E., Lanoot, B., De Cleene, M., Mew, T. W., and Swings, J. 2001. Bacterial populations associated with rice seed in the tropical environment. Phytopathology 91:282-292.

12. Escalon, A., Javegny, S., Verniere, C., Noel, L. D., Vital, K., Poussier, S., Hajri, A., Boureau, T., Pruvost, O., Arlat, M., and Gagnevin, L. 2013. Variations in type III effector repertoires, pathological phenotypes and host range of Xanthomonas citri pv. citri pathotypes. Mol. Plant Pathol. 14:483-496.

13. Gonzalez, C., Szurek, B., Manceau, C., Mathieu, T., Sere, Y., and Verdier, V. 2007. Molecular and pathotypic characterization of new Xanthomonas oryzae strains from West Africa. Mol. Plant-Microbe Interact. 20:534546.

14. Grau, J., Wolf, A., Reschke, M., Bonas, U., Posch, S., and Boch, J. 2013. Computational predictions provide insights into the biology of TAL effector target sites. PLoS Comput. Biol. 9:e1002962.

15. Guo, W., Cui, Y. P., Li, Y. R., Che, Y. Z., Yuan, L., Zou, L. F., Zou, H. S., and Chen, G. Y. 2012. Identification of seven Xanthomonas oryzae pv. oryzicola genes potentially involved in pathogenesis in rice. Microbiology 158:505-518.

16. Hajri, A., Brin, C., Hunault, G., Lardeux, F., Lemaire, C., Manceau, C., Boureau, T., and Poussier, S. 2009. A "repertoire for repertoire" hypothesis: repertoires of type three effectors are candidate determinants of host specificity in Xanthomonas. PLoS One 4:e6632.

17. Hajri, A., Brin, C., Zhao, S., David, P., Feng, J. X., Koebnik, R., Szurek, B., Verdier, V., Boureau, T., and Poussier, S. 2011. Multilocus sequence analysis and type III effector repertoire mining provide new insights into the evolutionary history and virulence of Xanthomonas oryzae. Mol. Plant Pathol. 13:288-302.

18. Han, Q., Chen, Z., Deng, Y., Lan, T., Guan, H., Duan, Y., Zhou, Y., and Lin, M. W. R. W. 2008. Fine mapping of qBlsr5a, a QTL controlling resistance to bacterial leaf streak in rice. Acta Agron. Sin. 34:587-590.

19. Hopkins, C. M., White, F. F., Choi, S. H., Guo, A., and Leach, J. E. 1992. Identification of a family of avirulence genes from Xanthomonas oryzae pv. oryzae. Mol. Plant-Microbe Interact. 5:451-459.

20. Jones, R. K., Barnes, L. W., Gonzalez, C. F., Leach, J. E., Alvarez, A. M., and Benedict, A. A. 1989. Identification of low virulence strains of Xanthomonas campestris pv. oryzae from rice in the United States. Phytopathology 79:984-990.

21. Kang, M. J., Shim, J. K., Cho, M. S., Seol, Y. J., Hahn, J. H., Hwang, D. J., and Park, D. S. 2008. Specific detection of Xanthomonas oryzae pv. oryzicola in infected rice plant by use of PCR assay targeting a membrane fusion protein gene. J. Microbiol. Biotechnol. 18:1492-1495. 
22. Karganilla, A., Paris-Natural, M., and Ou, S. 1973. A comparative study of culture media for Xanthomonas oryzae. Philipp. Agric. 57:141-152.

23. Lang, J. M., Hamilton, J. P., Diaz, M. G. Q., Van Sluys, M. A., Burgos, M. A. G., Vera Cruz, C. M., Buell, C. R., Tisserat, N. A., and Leach, J. E. 2010. Genomics-based diagnostic marker development for Xanthomonas oryzae pv. oryzae and X. oryzae pv. oryzicola. Plant Dis. 93:311-319.

24. Lee, B. M., Park, Y. J., Park, D. S., Kang, H. W., Kim, J. G., Song, E. S., Park, I. C., Yoon, U. H., Hahn, J. H., Koo, S., Lee, G. B., Kim, H., Park, H. S., Yoon, K. O., Kim, J. H., Jung, C. H., Koh, N. H., Seo, J. S., and Go, S. J. 2005. The genome sequence of Xanthomonas oryzae pathovar oryzae KACC10331, the bacterial blight pathogen of rice. Nucleic Acids Res. 33:577-586.

25. Makino, S., Sugio, A., White, F., and Bogdanove, A. J. 2006. Inhibition of resistance gene-mediated defense in rice by Xanthomonas oryzae pv. oryzicola. Mol. Plant-Microbe Interact. 19:240-249.

26. Moffett, M., and Croft, B. 1983. Xanthomonas. Pages 189-228 in: Plant Bacterial Diseases. A Diagnostic Guide. P. Fahy and G. Persley, eds. Academic Press, Sydney, Australia.

27. Muller, C., Cramer, W., Hare, W. L., and Lotze-Campen, H. 2011. Climate change risks for African agriculture. Proc. Natl. Acad. Sci. USA 108:4313-4315.

28. Nino-Liu, D. O., Ronald, P. C., and Bogdanove, A. J. 2006. Xanthomonas oryzae pathovars: model pathogens of a model crop. Mol. Plant Pathol. 7:303-324.

29. Ochiai, H., Inoue, V., Takeya, M., Sasaki, A., and Kaku, H. 2005. Genome sequence of Xanthomonas oryzae pv. oryzae suggests contribution of large numbers of effector genes and insertion sequences to its race diversity. JARQ 39:275-287.

30. Ou, S. H. 1985. Pages 247-256 in: Rice Diseases, 2nd ed. Commonwealth Mycological Institute, Kew, England.

31. Pan, R. W., Zou, W. C., Xu, D. G., Pan, R. Q., and Ji, C. Y. 2010. Genotypic and pathotypic diversity of the Xanthomonas oryzae pv. oryzicola in southern China. (Abstr.) Phytopathology 100:S96.

32. Parkinson, H., Kapushesky, M., Kolesnikov, N., Rustici, G., Shojatalab, M., Abeygunawardena, N., Berube, H., Dylag, M., Emam, I., Farne, A., Holloway, E., Lukk, M., Malone, J., Mani, R., Pilicheva, E., Rayner, T. F., Rezwan, F., Sharma, A., Williams, E., Bradley, X. Z., Adamusiak, T., Brandizi, M., Burdett, T., Coulson, R., Krestyaninova, M., Kurnosov, P., Maguire, E., Neogi, S. G., Rocca-Serra, P., Sansone, S. A., Sklyar, N., Zhao, M., Sarkans, U., and Brazma, A. 2009. ArrayExpress update--from an archive of functional genomics experiments to the atlas of gene expression. Nucleic Acids Res. 37:D868-872.

33. Parkinson, N., Aritua, V., Heeney, J., Cowie, C., Bew, J., and Stead, D. 2007. Phylogenetic analysis of Xanthomonas species by comparison of partial gyrase B gene sequences. Int. J. Syst. Evol. Microbiol. 57:28812887.

34. Rademaker, J. L., Hoste, B., Louws, F. J., Kersters, K., Swings, J., Vauterin, L., Vauterin, P., and de Bruijn, F. J. 2000. Comparison of AFLP and rep-PCR genomic fingerprinting with DNA-DNA homology studies: Xanthomonas as a model system. Int. J. Syst. Evol. Microbiol. 50:665677.

35. Raymundo, A. K., Briones, A. M. J., Ardales, E. Y., Perez, M. T., Fernandez, L. C., Leach, J. F., Mew, T. W., Ynalvez, M. A., McLaren, C. G., and Nelson, R. J. 1999. Analysis of DNA polymorphism and virulence in Philippine strains of Xanthomonas oryzae pv. oryzicola. Plant Dis. 83:434-440.

36. Reimers, P. J., and Leach, J. E. 1991. Race-specific resistance to Xanthomonas oryzae pv. oryzae conferred by bacterial blight resistance gene $\mathrm{Xa}-10$ in rice (Oryza sativa) involves accumulation of a lignin-like substance in host tissues. Physiol. Mol. Plant Pathol. 38:39-55.

37. Ryba-White, M., Notteghem, J. L., and Leach, J. E. 1995. Comparison of Xanthomonas oryzae pv. oryzae strains from Africa, North America, and Asia by restriction fragment length polymorphism analysis. Int. Rice Res. 20:25-26.

38. Salzberg, S. L., Sommer, D. D., Schatz, M. C., Phillippy, A. M., Rabinowicz, P. D., Tsuge, S., Furutani, A., Ochiai, H., Delcher, A. L., Kelley, D., Madupu, R., Puiu, D., Radune, D., Shumway, M., Trapnell, C., Aparna, G., Jha, G., Pandey, A., Patil, P. B., Ishihara, H., Meyer, D. F., Szurek, B., Verdier, V., Koebnik, R., Dow, J. M., Ryan, R. P., Hirata, H., Tsuyumu, S., Won Lee, S., Seo, Y. S., Sriariyanum, M., Ronald, P. C., Sonti, R. V., Van Sluys, M. A., Leach, J. E., White, F. F., and Bogdanove, A. J. 2008. Genome sequence and rapid evolution of the rice pathogen Xanthomonas oryzae pv. oryzae PXO99A. BMC Genomics 9:204.
39. Sigee, D. C. 1993. X-Ray Microanalysis in Biology: Experimental Techniques and Applications. Cambridge University Press, Cambridge.

40. Singh, D. V., Banerjee, A. K., Kishun, R., and Abidi, A. B. 1980. Effect of bacterial leaf streak on the quantitative and qualitative characters of rice. Indian J. Mycol. Plant Pathol. 10:67-68.

41. Streubel, J., Pesce, C., Hutin, M., Koebnik, R., Boch, J., and Szurek, S. 2013. Five phylogenetically close rice SWEET genes confer TAL effectormediated susceptibility to Xanthomonas oryzae pv. oryzae. New Phytol. doi: $10.1111 / \mathrm{nph} .12411$

42. Swings, J., Van Den Mooter, M., Vauterin, L., Hoste, B., Gillis, M., Mew, T. W., and Kersters, K. 1990. Reclassification of the causal agents of bacterial blight Xanthomonas campestris pathovar oryzae and bacterial leaf streak Xanthomonas campestris pathovar oryzicola of rice as pathovars of Xanthomonas oryzae new species Ex Ishiyama 1922 Revived Name. Int. J. Syst. Bacteriol. 40:309-311.

43. Tamura, K., Peterson, D., Peterson, N., Stecher, G., Nei, M., and Kumar, S. 2010. MEGA5: molecular evolutionary genetics analysis using maximum likelihood, evolutionary distance, and maximum parsimony methods. Mol. Biol. Evol. 28:2731-2739.

44. Tang, D., Wu, W., Li, W., Lu, H., and Worland, A. J. 2000. Mapping of QTLs conferring resistance to bacterial leaf streak in rice. Theor. Appl. Genet. 101:286-291.

45. Traore, O., Pinel-Galzi, A., Sorho, F., Sarra, S., Rakotomalala, M., Sangu, E., Kanyeka, Z., Sere, Y., Konate, G., and Fargette, D. 2009. A reassessment of the epidemiology of Rice yellow mottle virus following recent advances in field and molecular studies. Virus Res. 141:258-267.

46. Triplett, L. R., Hamilton, J. P., Buell, C. R., Tisserat, N. A., Verdier, V., Zink, F., and Leach, J. E. 2011. Genomic analysis of Xanthomonas oryzae isolates from rice grown in the United States reveals substantial divergence from known $X$. oryzae pathovars. Appl. Environ. Microbiol. 77:3930-3937.

47. Verdier, V., Triplett, L. R., Hummel, A. W., Corral, R., Cernadas, R. A., Schmidt, C. L., Bogdanove, A. J., and Leach, J. E. 2012. Transcription activator-like (TAL) effectors targeting OsSWEET genes enhance virulence on diverse rice (Oryza sativa) varieties when expressed individually in a TAL effector-deficient strain of Xanthomonas oryzae. New Phytol. 196:1197-1207.

48. Verdier, V., Vera Cruz, C., and Leach, J. E. 2012. Controlling rice bacterial blight in Africa: needs and prospects. J. Biotechnol. 159:320328.

49. Wonni, I., L. Ouedraogo, L., and Verdier, V. 2011. First report of bacterial leaf streak caused by Xanthomonas oryzae pv. oryzicola on rice in Burkina Faso. Plant Dis. Rep. 1:72.

50. Xie, G., Sun, S., Wang, G., Zhu, X., Chen, J., Ye, Y., Feng, Z., and Liang, M. 1990. Studies on rice seed inspection of Xanthomonas campestris pv. oryzicola: immunoradiometric assay. Chin. J. Rice Sci. 3:127-132.

51. Xie, G. L., and Mew, T. W. 1998. A leaf inoculation method for detection of Xanthomonas oryzae pv. oryzicola from rice seed. Plant Dis. 82:10071011.

52. Young, J. M., Park, D. C., Shearman, H. M., and Fargier, E. 2008. A multilocus sequence analysis of the genus Xanthomonas. Syst. Appl. Microbiol. 31:366-377.

53. Yu, Y., Streubel, J., Balzergue, S., Champion, A., Boch, J., Koebnik, R., Feng, J., Verdier, V., and Szurek, B. 2011. Colonization of rice leaf blades by an African strain of Xanthomonas oryzae pv. oryzae depends on a new TAL effector that induces the rice nodulin-3 Os 11N3 gene. Mol. PlantMicrobe Interact. 24:1102-1113.

54. Zhao, B., Ardales, E. Y., Raymundo, A., Bai, J., Trick, H. N., Leach, J. E., and Hulbert, S. H. 2004. The avrRxol gene from the rice pathogen Xanthomonas oryzae pv. oryzicola confers a nonhost defense reaction on maize with resistance gene Rxo1. Mol. Plant-Microbe Interact. 17:771779 .

55. Zhao, B., Lin, X., Poland, J., Trick, H., Leach, J., and Hulbert, S. 2005 b. A maize resistance gene functions against bacterial streak disease in rice. Agric. Sci. 102:15383-15388.

56. Zhao, S., Poulin, L., Rodriguez, R. L., Serna, N. F., Liu, S. Y., Wonni, I., Szurek, B., Verdier, V., Leach, J. E., He, Y. Q., Feng, J. X., and Koebnik, R. 2012. Development of a variable number of tandem repeats typing scheme for the bacterial rice pathogen Xanthomonas oryzae pv. oryzicola. Phytopathology 102:948-956.

57. Zou, H. S., Yuan, L., Guo, W., Li, Y. R., Che, Y. Z., Zuo, D. E., and Chen, G. Y. 2011. Construction of a Tn5-tagged mutant library of Xanthomonas oryzae pv. oryzicola as an invaluable resource for functional genomics. Curr. Microbiol. 62:908-916. 